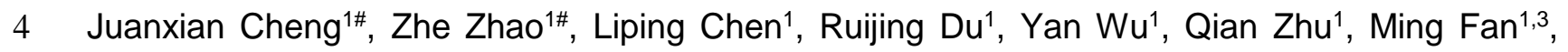

5 Xiaotao Duan ${ }^{4^{*}}$, Haitao $\mathrm{Wu}^{1,2,3,5^{*}}$

\section{Loss of O-GIcNAcylation on MeCP2 Thr 203 Leads to Neurodevelopmental Disorders}

${ }^{1}$ Department of Neurobiology, Beijing Institute of Basic Medical Sciences, 100850 Beijing, China;

${ }^{2}$ Chinese Institute for Brain Research, 102206 Beijing, China.

${ }^{3}$ Key Laboratory of Neuroregeneration, Co-innovation Center of Neuroregeneration, Nantong

University, Nantong, 226019 Jiangsu Province, China

${ }^{4}$ State Key Laboratory of Toxicology and Medical Countermeasures, Beijing Institute of Pharmacology and Toxicology, 100850 Beijing, China

${ }^{5}$ Lead Contact

\# These authors contributed equally to this work

${ }^{*}$ Correspondence:

Haitao Wu, Department of Neurobiology, Beijing Institute of Basic Medical Sciences, Taiping

Rd.27, Haidian District, Beijing, 100850, China

Tel: +86-10-66931363; Fax: +86-10-68213039; E-mail: wuht@bmi.ac.cn

Xiaotao Duan, State Key Laboratory of Toxicology and Medical Countermeasures, Beijing

Institute of Pharmacology and Toxicology, Taiping Rd.27, Haidian District, Beijing, 100850,

China

Tel: +86-10-66874710; E-mail: xduan@ncba.ac.cn

5

Running title: MeCP2 T203 O-GlcNAc Regulates Neural Development 


\section{Abstract}

Mutations of the X-linked methyl-CpG-binding protein 2 (MECP2) gene in humans are responsible for most cases of Rett syndrome (RTT), an X-linked progressive neurological disorder.

31 While genome-wide screens in clinical trials reveal several putative RTT-associated mutations on $M E C P 2$, their causative relevance regarding the functional regulation of MeCP2 on the etiologic

33 sites at the protein level require more evidence. In this study, we demonstrate that MeCP2 is

34 dynamically modified by O-linked- $\beta-N$-acetylglucosamine (O-GlcNAc) at threonine 203 (T203), an

35 etiologic site in RTT patients. Disruption of the O-GlcNAcylation of MeCP2 specifically at T203

36 impairs dendrite development and spine maturation in cultured hippocampal neurons, and

37 disrupts neuronal migration, dendritic spine morphogenesis and dysfunction of synaptic

38 transmission in the developing and juvenile mouse cerebral cortex. Mechanistically, genetic

39 disruption of O-GIcNAcylation at T203 on MeCP2 decreases neuronal activity-induced induction

40 of Bdnf transcription. Our study highlights the critical role of MeCP2 T203 O-GlcNAcylation in

41 neural development and synaptic transmission potentially via BDNF.

43 Keywords: MeCP2; O-GlcNAcylation; dendrite development; synaptic transmission; BDNF 


\section{INTRODUCTION}

Rett syndrome (RTT) is a severe X-linked neurodevelopmental disorder that preferentially occurred in females, with an approximate incidence of 1:10,000 1,2. Almost $90 \%$ of RTT cases are caused by methyl-CpG-binding protein 2 (MECP2) gene mutations such as missense, nonsense, insertion, deletion, and splice-site variations ${ }^{3,4,5}$, and loss of MeCP2 is closely related to the occurrence of RTT 1,6, 7, 8 . MeCP2 activity in normal central nervous system (CNS)

51 development and function is controlled by both precise expression levels ${ }^{9,10}$ and post-

52 translational modifications (PTMs). For example, neurons from Mecp2-null mutant mice have

53 smaller somas ${ }^{11,12}$, decreased dendritic complexity ${ }^{13,14,15}$ and dysfunction of synaptic plasticity 16, 17, 18. Gain-of-function MeCP2 by overexpression in transgenic mice or monkeys results in progressive neurological and psychiatric dysfunctions ${ }^{19,20}$. These genetic studies suggest that precise and dynamic expression of MeCP2 is critical to maintain normal brain development and 57 function.

In addition to expression level, PTMs on MeCP2 such as phosphorylation have been demonstrated as critical regulators of its role in dendritic growth, spine maturation, and activation of calcium-dependent brain-derived neurotrophic factor (BDNF) gene expression, suggesting that MeCP2 PTMs particularly impact neurodevelopment processes and activity-dependent

62 gene expression. ${ }^{21,22,23,24,25}$. Recently, the novel PTM O-GlcNAcylation has emerged as a

63 potent regulator of neurogenesis and synaptic plasticity ${ }^{26,27,28}$. O-GlcNAcylation is a highly 64 dynamic process ${ }^{29,30,31}$. O-GIcNAc transferase (OGT) catalyzes the addition of O-GlcNAc to 65 serine and threonine residues on intracellular proteins, whereas O-GlcNAcase (OGA) results in 66 the removal of O-GIcNAc modifications ${ }^{29,31,32}$. Previous studies have identified O-GlcNAcylation

67 as a potent modulator of neuronal differentiation ${ }^{26,33}$ and synaptic plasticity ${ }^{34}$. Interestingly, 68 MeCP2 was found to be O-GlcNAcylated and phosphorylated simultaneously in rat cortical 69 neurons ${ }^{35,36}$. However, despite the critical role of both O-GlcNAcylation and MeCP2 on neural 70 development and synaptic transmission, the physiological function and molecular mechanisms 
71 of human MeCP2 O-GlcNAcylation remain elusive.

In this report, we first used mass spectrometry (MS) to systematically identify OGlcNAcylation sites on mice, rats, and human MeCP2. We found that human MeCP2 T203, a site previously implicated in the pathogenesis of RTT 37,38, 39, was O-GlcNAcylated at relatively high levels at baseline compared to rodent species. Furthermore, we demonstrated the critical role of MeCP2 T203 O-GlcNAcylation in the regulation of dendrite outgrowth, dendritic spine morphogenesis and synaptic transmission both in vitro and in vivo. Mechanistic studies suggest that this may be due to the regulation of neuronal activity-induced induction of $B d n f$ transcription. Together, our results identified a previously unknown function of O-GlcNAcylated MeCP2 T203, which may be essential for understanding the molecular mechanisms behind the neuropathology

\section{RESULTS}

Identification of O-GIcNAcylation Sites on MeCP2

To understand whether MeCP2 is a highly conserved protein in different species, we first investigated the evolutionary divergence of the Mecp2 gene from humans and 25 additional representative species using phylogeny tree reconstruction. We found that the protein sequences of MeCP2 are conserved among most mammals, with distance values less than 0.1 , suggesting the sequence similarity between each pair of mammalian MeCP2 protein is over $90 \%$.

90 In contrast, sequence distances were much higher between mammalian and non-mammalian

91 species. This indicates that the protein sequence and function of MeCP2 have undergone 92 substantial changes during the evolution of vertebrates, but are relatively conserved in mammals 93 (Fig. 1A).

O-GIcNAcylation is highly dynamic and reversible in cellular systems. In order to better capture and concentrate O-GIcNAcylated MeCP2 for MS, HA-tagged MeCP2 in different species were co-expressed with OGT in HEK293T cells, and co-immunoprecipitated (co-IP) with anti-HA 
antibody-conjugated beads followed by in-gel trypsin digestion (Fig. S1A). The digested MeCP2 peptides were subjected to high-resolution mass spectrometry (MS, nanoLC-LTQ-CID/ETDOrbitrap) analysis (Table S1) (Fig. S1B-F). In addition to the O-GlcNAcylated sites on T434 and T444, which have been previously identified in rat MeCP2 ${ }^{35}$, 36, we mapped 11 novel OGlcNAcylation sites from mouse (mMeCP2), rat (rMeCP2) and human (hMeCP2) (Fig. 1B). To examine the potential relevance of these novel MeCP2 O-GIcNAc sites for neurodevelopmental disorders including RTT, we next compared them with the RTT disease-related MECP2 mutation sites reported from RettBASE ${ }^{37,38,39}$, a database that catalogues clinically-relevant MECP2 mutations. Interestingly, we found that three O-GlcNAcylated sites (S68, T203 and S204) were mutated in some RTT clinical cases ${ }^{40}$ (Table S2). In addition, human MeCP2 T436 (homologue of T434 in mMeCP2 and rMeCP2) O-GlcNAcylation may also be implicated, although no identified clinical cases have been associated with the mutation. Therefore, we mainly focused on four O-GIcNAcylated sites of human MeCP2 including S68, T203, S204 and T436 in the following assays (Fig. 1C-F). Of these sites, additional analysis pointed to T203 as a particularly interesting target. We compared the MeCP2 O-GlcNAcylation sites identified in this study from eight different species to assess their conservation (Fig. 1G). While the majority of these sites are conserved among vertebrate animals, T203 O-GIcNAcylation was selectively conserved in primates such as human, macaque, chimpanzee, and marmoset, but not in rodents. Given that MeCP2 T203 is an etiologic site in RTT patients, the T203 site may carry particular evolutionary functional significance in the brains of high primates.

\section{MeCP2 is O-GIcNAcylated at T203 by Direct Interaction with OGT}

The above MS results indicate that MeCP2 may serve as a new protein substrate of OGT (Fig. S1G). To test this hypothesis, we first investigated the interaction between MeCP2 and OGT, and to identify the MeCP2-binding domain within OGT. Endogenous MeCP2 from mouse whole brain lysates were co-immunoprecipitated with native OGT (Fig. 2A). Reciprocal assays in mouse 
brain lysates showed that OGT also co-immunoprecipitated with MeCP2 (Fig. 2B), strongly suggesting physiological interaction in vivo. To further explore if MeCP2 and OGT bind via direct protein-protein interaction, we performed an in vitro GST protein pull-down assay using purified GST-fused OGT and human MeCP2 protein. GST-fused OGT, but not GST alone, was able to pull down MeCP2 and vice versa, suggesting direct binding with each other (Fig. 2C and D). To reveal the reciprocal binding domains supporting the interaction of OGT with MeCP2, we first generated various human OGT deletion mutants. Co-IP assays showed that deletion of the entire

130 N-terminal TPR domain (aa 2-465) of OGT completely disrupted the interaction between OGT 131 and MeCP2, suggesting the TPR domain is necessary (Fig. 2E and F). Next, domain mapping 132 analysis showed that deletion of 4-6 TPR (aa 114-214), 7-9 TPR (aa 215-316) or 10-13.5 TPR 133 (aa 317-465), had no effects on the interaction between OGT and MeCP2 (Fig. 2E and F). In 134 contrast, deletion of the 1-3 TPR domain (aa 2-113) of OGT significantly abolished the binding 135 (Fig. 2E-H), suggesting OGT directly interacts with MeCP2 via its N-terminal 1-3 TPR domain. 136 Moreover, in order to identify critical OGT-binding domains on MeCP2, we used MeCP2 deletion 137 mutants in co-IP binding experiments with OGT, and found that both NTD and CTD domains of MeCP2 are necessary (Fig. S2).

To further study the mechanisms of MeCP2 O-GlcNAcylation by OGT in vivo, we setup a well-established chemoenzymatic labelling approach ${ }^{41}$ to detect MeCP2 O-GIcNAcylation from whole brain lysates (Fig. 3A). O-GIcNAc-modified proteins from mouse brain lysates were 142 enzymatically labelled with an azido-N-acetylgalactosamine sugar and biotinylated via $\mathrm{Cu}(\mathrm{I})-$ 143 mediated [3+2] azide-alkyne cycloaddition (CuACC) chemistry, which were captured with 144 streptavidin agarose beads, and subsequently immunoblotted with an antibody against MeCP2 145 (Fig. 3A). As shown in Fig. 3B, O-GlcNAcylation occurs in endogenous MeCP2 in wild type (WT) 146 mice in vivo, and much stronger O-GlcNAcylation modification of MeCP2 was detected in 147 overexpression of MECP2 transgenic mice ${ }^{19}$ compared with WT mice. The above results suggest 148 direct protein-protein binding between MeCP2 and OGT that results in O-GIcNAcylation of MeCP2 
(Fig. 2). To further confirm that OGT catalyzes MeCP2 upon direct protein binding, we used co-IP assay to demonstrate that ectopic GFP-tagged hMeCP2 was O-GlcNAcylated in the presence of OGT in transfected HEK293T cells (Fig. 3C). Additionally, in vitro O-GlcNAcylation assay further confirmed that His-tagged hMeCP2 was directly O-GlcNAcylated by GST-tagged enzyme domain of OGT (323-1041) ${ }^{42}$ (Fig. 3D).

Because our MS results indicated several O-GIcNAcylation sites in hMeCP2 with potential relevance to RTT (Fig. 1 and Fig. S1) we wanted to further identify major functional O-

156 GlcNAcylation activity among these sites. To test this, MeCP2 S68A, T203M, S204A, T436A, and 157 quadruple site mutation to alanine (4 Muts) were generated. We found that the level of O158 GlcNAcylation was significantly reduced in the T203M and 4 Muts mutant variations in vitro (Fig. 159 3E and F) and in vivo (Fig. 3G and H). However, changes in O-GlcNAcylation were insignificant 160 for other MeCP2 mutants, suggesting that the T203 site is the predominant O-GlcNAcylation site 161 on human MeCP2. Of note, the T203 site has previously been implicated in the pathogenesis of 162 RTT. Interestingly, MeCP2-4Muts still showed a weak level of O-GlcNAcylation, which further 163 supports our MS results showing multiple O-GlcNAcylation sites on MeCP2 (Fig. 1 and Fig. S1).

To interrogate the reversibility of MeCP2 O-GIcNAcylation, we introduced GST-tagged OGA $165(31-624)$ recombinant protein, which removes O-GIcNAc modifications. OGA almost completely removed the O-GlcNAcylation on MeCP2 in vitro (Fig. 3I-L), implying that MeCP2 O-

167 GlcNAcylation is a reversible and dynamic process.

\section{T203 O-GIcNAcylation is Required for Dendrite Development in Cultured Neurons}

To understand the functional importance of MeCP2 O-GIcNAcylation, we next examined its

171 impact on neural development. Overexpression of MeCP2 inhibits dendritic growth of

172 hippocampal neurons ${ }^{43,44}$, and MECP2 transgenic mice also appears to have a progressive 173 neurological and neurobehavioral disorders ${ }^{19,44,45}$. If MeCP2 O-GlcNAcylation is involved in these 174 aberrant neural developmental physiology, we reasoned that mutating O-GlcNAc sites in an 
MeCP2 overexpression system may rescue the above observed defects. Thus, for our next experiments, we employed MeCP2 overexpression as an experimental model to test the significance of MeCP2 T203 O-GlcNAcylation for neural development.

First, we compared the morphological differences of dendritic branches of cultured mouse hippocampal neurons using anti-MAP2 immunofluorescent staining after infection with the indicated LV-GFP control or LV-hMeCP2 ectopic expression lentivirus (Fig. S3A). The total length of dendrites was significantly inhibited by overexpressing ectopic hMeCP2 compared with cells

182 infected with the LV-GFP control virus $(207.4 \pm 7.85 \mu \mathrm{m}$ in LV-hMeCP2 and $294.2 \pm 10.17 \mu \mathrm{m}$ in

183 LV-GFP control, $\mathrm{p}<0.001, \mathrm{n}=62$ and 67 neurons, respectively). However, overexpression of 184 hMeCP2 T203M or hMeCP2-4Muts mutant had no significant effect on the length of total dendrite compared with LV-GFP control neurons $(269.0 \pm 9.58 \mu \mathrm{m}$ in LV-hMeCP2 T203M and 263.6 \pm 8.81 $\mu \mathrm{m}$ in LV-hMeCP2-4Muts, respectively; $\mathrm{p}=0.1348$ and $0.0829, \mathrm{n}=63$ and 56 neurons, respectively) (Fig. S3B and C). In stark contrast, overexpression of the hMeCP2 O-GlcNAc mutant hMeCP2 S68A, S204A or T436A all had similar inhibitory effects on dendrite development as WT hMeCP2 ( $p<0.001, n=70,60$ and 63 neurons, respectively). These results suggest that T203M, but not S68A, S204A or T436A, specifically rescues the aberrant dendritic morphology seen with hMeCP2 overexpression. In addition, we also quantified the length of primary and secondary

192 dendritic branches after overexpression of the indicated ectopic hMeCP2. As shown in Fig. S3D 193 and E, overexpression of WT hMeCP2 significantly inhibited the length of both primary and 194 secondary branches compared with LV-GFP controls (162.0 $\pm 6.02 \mu \mathrm{m}$ in LV-hMeCP2 and 214.4 $195 \pm 6.56 \mu \mathrm{m}$ in LV-GFP control for primary branches, $\mathrm{p}<0.001, \mathrm{n}=62$ neurons; $31.3 \pm 3.70 \mu \mathrm{m}$ in 196 LV-hMeCP2 and $71.4 \pm 5.87 \mu \mathrm{m}$ in LV-GFP control for secondary branches, $\mathrm{p}<0.001, \mathrm{n}=27$ 197 neurons). Overexpression of hMeCP2 S68A, S204A and T436A also significantly decreased the 198 length of primary branches ( $p<0.001, n=58,50$ and 63 neurons, respectively), but 199 overexpression of MeCP2 T203M or MeCP2-4Muts mutant had no obvious effect on the length 200 of both primary and secondary branches (Fig. S3D and E). The rescue effect seen with MeCP2 
T203M and MeCP2-4Muts seen to be relatively specific, because overexpression of either S68A, S204A or T436A mutant inhibited neuronal dendrite development similar to overexpressed WT hMeCP2 (Fig. S3B-E). Together, these results indicate that O-GlcNAcylation of hMeCP2 T203 critically underlies the dendritic deficits observed with overexpression of ectopic hMeCP2 in cultured hippocampal neurons, and mutating T203 rescues these deficits in dendritic length and branching.

To further illustrate the significance of MeCP2 O-GlcNAcylation in neural development, we tested whether T203 O-GlcNAcylation had any effects on dendritic spine morphogenesis in cultured hippocampal neurons. A lentiviral-based rescue (LEMPRA) construct was used to exogenously introduce Flag-tagged shRNA-resistant human MECP2 to replace endogenous

211 mouse Mecp2, the expression of which was specifically knocked down by shRNA ${ }^{24}$ (Fig. 4A).

212 Cultured hippocampal neurons from E17.5 mouse embryos were infected with the indicated 213 lentivirus at DIV 7, then fixed at DIV 14 for immunofluorescent staining with the postsynaptic 214 marker PSD-95 antibody (Fig. 4B) to evaluate the formation of dendritic spines (Fig. 4C). Efficient 215 knockdown of endogenous mMeCP2 and expression of equivalent levels of exogenous hMeCP2 216 were confirmed by Western blot analysis (Fig. S4). Consistent with previous reports showing 217 smaller somas in Mecp2-null neurons ${ }^{11,12}$, quantification revealed that the area of soma in 218 shMeCP2 (mMeCP2-) neurons is dramatically reduced compared with FUGW-GFP control 219 neurons $\left(129.1 \pm 8.18 \mu \mathrm{m}^{2}\right.$ in mMeCP2-, and $211.5 \pm 18.7 \mu \mathrm{m}^{2}$ in FUGW-GFP control, $p<0.01, n$ $220=22$ and 15 neurons, respectively). Importantly, exogenous expression of WT hMeCP2 or $221 \mathrm{hMeCP} 2 \mathrm{~T} 436 \mathrm{~A}\left(203.4 \pm 16.71 \mu \mathrm{m}^{2}\right.$ in mMeCP2-/hMeCP2+ and $200.9 \pm 16.04 \mu \mathrm{m}^{2}$ in mMeCP2222 /hMeCP2 T436A+, $\mathrm{p}=0.9904$ and 0.9875, respectively, compared with FUGW-GFP control, $\mathrm{n}=$ 22320 and 9 neurons, respectively), but not hMeCP2 T203M or hMeCP2-2Muts mutants (162.9 \pm $2248.43 \mu^{2}$ in mMeCP2-/hMeCP2 T203M+ and $143.9 \pm 9.49 \mu \mathrm{m}^{2}$ in mMeCP2-/hMeCP2-2Muts+, p $225=0.0348$ and 0.0174 , respectively, compared with FUGW-GFP control, $\mathrm{n}=23$ and 9 neurons, 226 respectively), efficiently rescued the smaller soma deficiency in mMeCP2- neurons (Fig. 4D). 
In agreement with previous reports in Mecp2-null mice 9, 46, 47, we found that the density of PSD-95 puncta was significantly decreased after knocking down endogenous MeCP2 (8.2 \pm 0.53 $/ 10 \mu \mathrm{m}$ in mMeCP2- and $17.0 \pm 0.56 / 10 \mu \mathrm{m}$ in FUGW-GFP control, $\mathrm{p}<0.001, \mathrm{n}=10$ neurons). Both exogenously expressed Flag-tagged WT hMeCP2 and hMeCP2 T436A mutants efficiently rescued the decrease in PSD-95 puncta after MeCP2 knockdown $(16.9 \pm 0.39 / 10 \mu \mathrm{m}$ in $\mathrm{mMeCP} 2-/ \mathrm{hMeCP} 2+$ and $16.5 \pm 0.27 / 10 \mu \mathrm{m}$ in mMeCP2-/hMeCP2 T436A+, $\mathrm{p}=0.9997$ and 0.8970, respectively, compared with FUGW-GFP control, $n=10)$. Interestingly, in stark contrast, exogenously expressed hMeCP2 T203M and hMeCP2-2Muts both showed significantly decreased number of PSD-95 puncta compared with FUGW-GFP control neurons $(8.4 \pm 0.52 / 10$ $\mu \mathrm{m}$ in $\mathrm{mMeCP} 2-/ \mathrm{hMeCP} 2 \mathrm{~T} 203 \mathrm{M}+$ and $8.5 \pm 0.37 / 10 \mu \mathrm{m}$ in mMeCP2-/hMeCP2-2Muts+, $\mathrm{p}<0.01$ and $p<0.01$, respectively, $n=10$ neurons) (Fig. 4E), demonstrating an inability to rescue MeCP2 knockdown-induced decrease in PSD-95. In addition, we also analyzed the average size of each PSD-95 puncta within the dendritic spines. We found that the PSD-95 puncta size was significantly decreased in mMeCP2- neurons compared to FUGW-GFP control neurons $(0.312 \pm$ $0.018 \mu \mathrm{m}^{2}$ in mMeCP2-, $\mathrm{n}=35 ; 0.432 \pm 0.026 \mu \mathrm{m}^{2}$ in FUGW-GFP control, $\left.\mathrm{n}=38 ; \mathrm{p}=0.001\right)$.

242 Ectopic expression of WT hMeCP2 and hMeCP2 T436A mutants both significantly rescued the decreased PSD-95-positive puncta size in mMeCP2- neurons to normal level compared with

244 FUGW-GFP control neurons $\left(0.466 \pm 0.027 \mu \mathrm{m}^{2}\right.$ in mMeCP2-/hMeCP2+, $\mathrm{n}=37 ; 0.428 \pm 0.027$ $245 \mu \mathrm{m}^{2}$ in mMeCP2-/hMeCP2 T436A+, $n=34 ; p=0.738$ and 0.999 , respectively). However, 246 exogenously expressed hMeCP2 T203M and hMeCP2-2Muts in mMeCP2- neurons both showed 247 significantly decreased size of PSD-95-positive puncta compared with FUGW-GFP control 248 neurons $\left(0.270 \pm 0.019 \mu \mathrm{m}^{2}\right.$ in $\mathrm{mMeCP} 2-/ \mathrm{hMeCP} 2 \mathrm{~T} 203 \mathrm{M}+, \mathrm{n}=37 ; 0.273 \pm 0.018 \mu \mathrm{m}^{2}$ in 249 mMeCP2-/hMeCP2-2Muts, $n=33 ; p<0.001$ and $p<0.001$, respectively) (Fig. 4F). Together, 250 these results suggest a requirement of T203 O-GlcNAcylation for dendritic spine morphogenesis. 251 Taken together, our results indicate that hMeCP2 T203 O-GlcNAcylation is essential for the 252 regulation of neurite outgrowth and dendritic spine morphogenesis in cultured hippocampal 
neurons. Moreover, T203 O-GlcNAcylation is also sufficient for the maintenance of neuronal soma

254 during neuronal differentiation in vitro.

\section{T203 O-GIcNAcylation Promotes Cortical Neuron Migration and Maturation in vivo}

To further understand the role of T203 O-GIcNAcylation in cortical development in vivo, we manipulated the expression of exogenous human MeCP2 in the developing mouse neocortex using the above-described lentiviral-based rescue (LEMPRA) plasmid by in utero electroporation

24, 48. Neuronal migration and dendritic differentiation are critical events in cortical construction.

261 Therefore, we analyzed the effects of T203 O-GlcNAcylation on neuronal migration and dendritic

262 spine morphogenesis at embryonic day 17.5 (E17.5) and postnatal day 15 (P15), respectively

263 (Fig. 5A). First, we found that loss-of-function of MeCP2 by shRNA-mediated knockdown

264 resulted in significant migration defects at E17.5 when compared with electroporation of only

265 GFP (Fig. 5B and C). To exclude possible off-target effects of the shRNA system, we showed

266 that the migration defects in MeCP2 knockdown neurons could be rescued by exogenous

267 expression of WT hMeCP2. However, exogenous expression of the hMeCP2 T203M mutant

268 could not rescue the migration defects in mMeCP2 knockdown neurons (Fig. 5B and C),

269 indicating T203 O-GIcNAcylation is required for proper neuronal migration in the embryonic 270 neocortex.

271 Previous studies has shown that the leading process (LP) branch is a critical determinant 272 for nuclear translocation during neuronal migration ${ }^{49}$. Therefore, we analyzed the morphology 273 and projection of LP in MeCP2 knockdown (mMeCP2-) and rescue neurons (mMeCP2$274 / \mathrm{hMeCP} 2+$ ). Interestingly, we found that the length of LP in confirmed mMeCP2- neurons is much 275 longer compared with FUGW-GFP control neurons (38.94 $\pm 1.07 \mu \mathrm{m}$ in mMeCP2- neurons and $27618.18 \pm 0.62 \mu \mathrm{m}$ in FUGW-GFP control neurons, $\mathrm{p}<0.001, \mathrm{n}=35$ and 37 , respectively). 277 Moreover, the abnormal LP branches in confirmed mMeCP2- neurons can be significantly 278 rescued by exogenous expression of WT hMeCP2, but not hMeCP2 T203M mutant (19.98 \pm 

neurons, $p=0.4528$ and $p<0.001$, respectively, compared with FUGW-GFP control, $n=36$ and 35, respectively) (Fig. 5D). Collectively, these results indicate that the abnormally developed LP branches may be responsible for the migration defects in mMeCP2- neurons, and that hMeCP2 T203 O-GlcNAcylation is critical for maintaining normal LP branch morphology and the navigation of migrating neuronal precursors in vivo.

Dendritic spines have been showed to be abnormal in the cerebral cortex in RTT patients and Mecp2-null mice ${ }^{11,13,47,50}$. Thus, we next asked whether MeCP2 T203 O-GlcNAcylation is essential for postnatal dendritic spine morphogenesis in vivo. First, we analyzed both dendrite and dendritic spine morphology in Layer II/III cortical projection neurons on P15 mice, which had been manipulated by in utero electroporation as described above on E13.5 (Fig. 5A). GFP fluorescence was used to trace the morphology of entire neurons, including the apical and basal dendritic arbors. Initial analysis did not find striking differences based on gross morphology of the dendrites including polarity and dendritic orientation in individual groups of electroporated neurons (Fig. 5E). Next, we imaged apical and basal dendritic segments at high magnification for each groups of electroporated neurons (Fig. 5E), and found that the density of both apical and basal spines were significantly decreased in mMeCP2- neurons compared with FUGW-GFP $297 / \mu \mathrm{m}$ and $1.37 \pm 0.054 / \mu \mathrm{m}$ in FUGW-GFP control neurons, $\mathrm{p}=0.0045$ and 0.013 , respectively, $\mathrm{n}$ $298=13)($ Fig. $5 F$ and G). Meanwhile, the spine formation defects in mMeCP2- neurons can be 299 significantly rescued by ectopic expression of WT hMeCP2, but not by hMeCP2 T203M mutant $300 \quad(1.415 \pm 0.057 / \mu \mathrm{m}$ and $1.36 \pm 0.052 / \mu \mathrm{m}$ in $\mathrm{mMeCP} 2-/ \mathrm{hMeCP} 2+$ neurons, and $0.831 \pm 0.052$ $301 / \mu \mathrm{m}$ and $0.85 \pm 0.059 / \mu \mathrm{m}$ in mMeCP2-/hMeCP2 T203M+ neurons, $p=0.99$ and $p<0.001$, 302 respectively, compared with FUGW-GFP control neurons, $n=17$ ) (Fig. 5F and $G$ ).

303 Dendritic spines are morphologically heterogeneous within the neocortex, and these 304 differences between spines reflect functional viability and pathological states ${ }^{51}$. To interrogate 
the effect of hMeCP2 T203 O-GlcNacylation on spine morphology, we next classified spine shapes into thin, mushroom, and stubby categories as previously defined ${ }^{52}$. We found significantly fewer mushroom spines in mMeCP2- neurons at P15, compared with FUGW-GFP control neurons $(38.79 \pm 1.89 \%$ in mMeCP2- neurons and $51.18 \pm 2.03 \%$ in FUGW-GFP control neurons, $p=0.001, n=17$ and 38 , respectively) (Fig. 5H). In contrast, no changes in spine morphology were observed in mMeCP2-/hMeCP2+ rescue neurons, compared with FUGW-GFP control neurons $(48.76 \pm 2.27 \%$ in mMeCP2-/hMeCP2+ neurons and $51.18 \pm 2.03 \%$ in FUGW-

312 GFP control neurons, $\mathrm{p}=0.9993, \mathrm{n}=18$ and 38, respectively). However, mMeCP2-/hMeCP2 313 T203M+ neurons had significantly fewer mushroom spines with relatively increased number of 314 stubby spines (41.96 $\pm 2.31 \%$ mushroom type and $48.50 \pm 3.11 \%$ stubby type in mMeCP2$315 /$ hMeCP2 T203M+ neurons; $51.18 \pm 2.03 \%$ mushroom type and $32.90 \pm 1.58 \%$ stubby type in 316 FUGW-GFP control neurons, $p=0.0465$ and $p<0.001$, respectively, $n=18$ and 38, respectively) 317 (Fig. 5H). Therefore, our results indicate that hMeCP2 T203 O-GIcNAcylation plays a critical role 318 in the regulation of dendritic spine morphogenesis. Notably, the features displayed in mMeCP2/hMeCP2 T203M+ cortical neurons nicely mimic the defects in dendrite and spine maturation and synaptogenesis in RTT patients ${ }^{11,13,50,53}$.

\section{T203 O-GIcNAcylation Regulates Excitatory Synaptic Transmission}

Previous studies have shown that shRNA-mediated MeCP2 knockdown affects homeostatic synaptic plasticity in hippocampal neurons ${ }^{54,55}$. Long-term potentiation (LTP) is also impaired at excitatory synapses of layers $\mathrm{II} / \mathrm{II}$ and $\mathrm{V}$ in the primary somatosensory cortex of Mecp2-null mutant mice ${ }^{56,57}$. However, the bases of these deficits remain unclear. To investigate whether synaptic transmission is regulated by hMeCP2 T203 O-GlcNAcylation, we treated neurons in layers II/III of the developing cortex with various LEMPRA constructs using in utero electroporation, and recorded from GFP-positive neurons on P21 (Fig. 6A). First, we recorded miniature excitatory postsynaptic currents (mEPSCs) in FUGW-GFP control and mMeCP2- projection neurons (Fig. 
6B and 6C). The mEPSC amplitudes remained similar between mMeCP2- neurons and FUGWGFP control neurons $(10.23 \pm 0.57 \mathrm{pA}$ in FUGW-GFP control, $\mathrm{n}=10$ neurons, and $11.24 \pm 0.26$ pA in mMeCP2-, $n=9$ neurons, $p=0.7859)$. Exogenous expression of WT or T203M mutant hMeCP2 in mMeCP2- neurons has on significant effect on the mEPSC amplitudes $(12.71 \pm 0.77$ pA in mMeCP2-/hMeCP2+, $\mathrm{n}=10$ neurons, and $12.59 \pm 0.88$ pA in mMeCP2-/hMeCP2 T203M+, $n=8$ neurons, compared with FUGW-GFP control neurons, $p=0.0939$ and 0.1793 , respectively) (Fig. 6D and 6E). In contrast, in agreement with the decreased dendritic spine density in mMeCP2- neurons (Fig. 4E, 5F and 5G) and previous report ${ }^{47}$, the mEPSC frequency significantly decreased from $3.00 \pm 0.26 \mathrm{~Hz}$ in FUGW-GFP control neurons to $1.62 \pm 0.11 \mathrm{~Hz}$ in mMeCP2- neurons ( $n=10$ and 9 neurons respectively, $p=0.0048$ ) (Fig. 6F and 6G). In addition, the reduced frequency of mEPSCs in mMeCP2- neurons was efficiently rescued by exogenous expression of WT hMeCP2, but not the hMeCP2 T203M mutant $(2.81 \pm 0.26 \mathrm{~Hz}$ in mMeCP2/hMeCP2+, $\mathrm{n}=10$ neurons, compared with FUGW-GFP control neurons, $\mathrm{p}>0.9999 ; 1.66 \pm 0.12$ $\mathrm{Hz}$ in mMeCP2-/hMeCP2 T203M+, $\mathrm{n}=8$ neurons, compared with FUGW-GFP control and mMeCP2-/hMeCP2+ neurons, $p=0.0093$ and 0.0259 , respectively) (Fig. 6F and 6G), further indicating that T203 O-GlcNAcylation is involved in the regulation of postsynaptic spine density and excitatory synaptic transmission in vivo.

Interestingly, both the amplitude and the frequency of miniature inhibitory postsynaptic currents (mIPSCs) were not significantly different between mMeCP2- neurons and FUGW-GFP control neurons (amplitude: $15.63 \pm 1.07 \mathrm{pA}$ in mMeCP2-, $\mathrm{n}=9$ neurons, and $15.01 \pm 1.15 \mathrm{pA}$ in

351 FUGW-GFP control, $n=10$ neurons, $p>0.9999$; frequency: $2.455 \pm 0.31 \mathrm{~Hz}$ in mMeCP2-, $n=9$ 352 neurons, and $2.426 \pm 0.31 \mathrm{~Hz}$ in FUGW-GFP control, $n=10$ neurons, $p>0.9999)$ (Fig. 6H-L). 353 Moreover, neither amplitude nor frequency of mIPSCs was changed in mMeCP2-/hMeCP2+ or 354 mMeCP2-/hMeCP2 T203M+ neurons compared with FUGW-GFP control neurons (amplitude: $35516.85 \pm 1.56 \mathrm{pA}$ in $\mathrm{mMeCP} 2-/ \mathrm{hMeCP} 2+, \mathrm{n}=9$ neurons, $\mathrm{p}>0.9999$, and $14.61 \pm 0.89 \mathrm{pA}$ in mMeCP2-/hMeCP2 T203M+, $n=11$ neurons, $p$ > 0.9999; frequency: $2.209 \pm 0.38$ Hz in mMeCP2- 
/hMeCP2+, $\mathrm{n}=9$ neurons, $\mathrm{p}>0.9999$, and $1.811 \pm 0.36 \mathrm{~Hz}$ in $\mathrm{mMeCP} 2-/ \mathrm{hMeCP} 2 \mathrm{~T} 203 \mathrm{M}+, \mathrm{n}=$ 11 neurons, $p=0.8159$ ) (Fig. $6 \mathrm{H}-\mathrm{L})$. Together, these results indicate that T203M O-GlcNAcylation may be essential for the establishment of excitatory, but not inhibitory, synaptic transmission during neurodevelopment in the cortex.

\section{Neuronal Activity-Induced Bdnf Transcription is Dependent on T203 O-GIcNAcylation}

MeCP2 regulates the expression of thousands of genes during neural development. One of the most important target genes is brain-derived neurotrophic factor (BDNF) ${ }^{21,22,24}$. Numerous studies have shown that BDNF is critical for dendritic spine morphogenesis, synaptic maturation, and synaptic plasticity ${ }^{58,59,60,61}$. Moreover, activity-induced release of BDNF modulates spine morphology in conjunction with spontaneous neurotransmitter release ${ }^{59}$. Therefore, we asked whether the impaired dendritic branching, spine morphogenesis and synaptic transmission observed in mMeCP2-/hMeCP2 T203M+ neurons were due to impaired Bdnf transcription. To evaluate the effect of T203 O-GlcNAcylation on neuronal activity-induced Bdnf transcription, primary mouse cortical neurons were dissected from E15.5 embryos and infected with various LEMPRA lentivirus constructions as described above (Fig. 4A) at DIV 3 for 4 days. We then added tetrodotoxin (TTX), a sodium channel selective blocker, into the culture medium at a final concentration of $1 \mu \mathrm{M}$ to block the production of action potentials for $12 \mathrm{hr}$. On DIV 8 , the cultured cortical neurons were treated with potassium chloride $(\mathrm{KCl})$ at a final concentration of $55 \mathrm{mM}$ for $5 \mathrm{hr}$ to trigger synchronous membrane depolarization $22,62,63$. The neurons were then harvested for RNA extraction and real time quantitative RT-PCR assay to investigate the transcription of MeCP2 target genes (Fig. 7A).

The transcription of total mouse Bdnf and its exon IV, but not exon VI, was dramatically enhanced by more than 4-fold in FUGW-GFP cortical neurons after $\mathrm{KCl}$ treatment $(p<0.001, \mathrm{n}$ = 3) (Fig. 7B-D). The upregulation of total Bdnf and exon IV mRNA transcription were significantly lower in mMeCP2- neurons compared with FUGW-GFP control neurons after treatment with $\mathrm{KCl}$ 
$383(p<0.001$ and $p<0.001$, respectively, $n=3)$ (Fig. 7B and C). Interestingly, the compromised 384 upregulation of Bdnf transcription in mMeCP2- neurons could be significantly rescued by exogenous expression of WT hMeCP2 and hMeCP2 T436A mutant $(p=0.1015$ and 0.1158 , respectively, $n=3)$, but not with hMeCP2 T203M or hMeCP2-2Muts mutants $(p<0.001$ and $p<$ 0.001 , respectively, $n=3$ ) (Fig. 7B and C). These results suggest that T203 O-GlcNAcylation of $\mathrm{hMeCP} 2$ is essential for neuronal activity-induced enhanced transcription of Bdnf, especially for exon IV, but not exon VI. In addition, we examined the transcription of Acta2, a downstream gene normally repressed by MeCP2 in cortical neurons ${ }^{43}$. We found that Acta2 mRNA level was significantly upregulated in mMeCP2- neurons in both absence and presence of $\mathrm{KCl}$ treatment conditions ( $p<0.001, n=3$ ) (Fig. 7E). Exogenous expression of WT hMeCP2 could significantly suppress the upregulated transcription of Acta2 in mMeCP2- neurons, but hMeCP2 T203M and hMeCP2-2Muts failed to reverse the transcriptional activation effect in mMeCP2- neurons ( $p>$ 0.9999 and $p<0.001$, respectively, $n=3$ ) (Fig. 7E), indicating that T203 O-GlcNAcylation of hMeCP2 is also required to suppress Acta2 transcription. These results suggest that hMeCP2 T203 O-GlcNAcylation is particularly important for activity-induced Bdnf expression in cortical neurons.

Finally, we performed chromatin immunoprecipitation (ChIP) analysis to quantify and compare the binding affinity of WT hMeCP2 and various hMeCP2 mutants to the mouse Bdnf IV 401 promoter in mouse Neuro2A cells (Fig. 7F and G). As expected, binding of MeCP2 to the Bdnf 402 IV promoter was significantly decreased in mMeCP2- cells (Fig. 7H). Exogenous expression of 403 either WT hMeCP2 or hMeCP2 T436A mutant dramatically rescued the binding deficiency in 404 mMeCP2- cells $(p=0.013$ and 0.5981 , respectively, compared with FUGW-GFP control cells, $n$ $405=3)$. In contrast, both hMeCP2 T203M and hMeCP2-2Muts failed to rescue the decreased 406 binding of Bdnf promoter IV in mMeCP2- cells ( $p=0.0004$ and $<0.001$, respectively, compared 407 with FUGW-GFP control cells, $\mathrm{n}=3$ ) (Fig. $7 \mathrm{H})$, suggesting decreased binding capacity of 408 hMeCP2 T203M, but not T436A, to Bdnf promoter IV. Taken together, these results indicate that 
MeCP2 directly controls mouse Bdnf transcription by binding to the promoter IV. T203 OGlcNAcylation on hMeCP2 is required to maintain Bdnf IV promoter binding, and therefore plays

411 a critical role in the upregulation of Bdnf transcription following neural activation.

\section{DISCUSSION}

Collectively, our results indicate that O-GIcNAcylation on hMeCP2 at T203 is critical for

415 dendritic growth, spine formation, baseline and induced synaptic transmission, and the 416 regulation of activity-induced Bdnf transcription. Using a LEMPRA-based shRNA system with 417 exogenous expression of various hMeCP2 O-GlcNAc site mutants, we demonstrated that O418 GlcNAcylation regulates MeCP2 activity during neurodevelopment, and pinpointed T203 as a 419 major novel PTM site for O-GIcNAc modification on hMeCP2. Given that T203 mutations were 420 previously implicated in clinical cases of RTT ${ }^{37,38,39}$, these results suggest that hMeCP2 T203 421 O-GIcNAcylation not only supports normal cortical neurodevelopment, but its deregulation may 422 contribute to the pathogenesis of RTT.

423 Mutations of the MECP2 gene are the most prevalent cause of RTT, and some mutated 424 sites are substrates for PTM $38,64,65,66$. This suggests that PTMs are a potentially crucial 425 component of maintaining normal MeCP2 function, and deregulation may result in neurodevelopmental pathologies. Extensive studies have previously shown that phosphorylation 428 function, suggesting diverse forms of regulation.

Of particular interest was the finding that rat MeCP2 proteins are O-GlcNAcylated ${ }^{35,36}$, but 430 its functional significance and mechanism was unclear. Previous results have implicated O431 GlcNAcylation in the pathogenesis of neurodegenerative diseases ${ }^{69,70}$. For example, the 432 imbalance of O-GIcNAcylation relative to phosphorylation on microtubule-associated protein Tau 433 may be highly involved in the pathogenesis of Alzheimer's disease ${ }^{71}$. In addition, OGT-mediated 434 O-GIcNAcylation modulates both the maturity and function of excitatory synapses in the 
435 developing brain ${ }^{72}$, and changes in OGT levels may contribute to synaptic plasticity deficits

436 during brain aging ${ }^{34}$. Here, our results further illustrate a novel role for O-GlcNAcylation as a 437 regulator of MeCP2 function during neurodevelopment. In particular, we identified T203 as a 438 critical site for the establishment and maintenance of normal dendritic and spine development 439 and synaptic transmission, in that exogenous expression of its mutant form T203M was not able 440 to rescue deficits seen with knockdown of mouse endogenous MeCP2, whereas rescue effects 441 were observed with other putative O-GlcNAcylation sites including S68, S204 and T436. The 442 unique role of T203 may be due to its location on the human MeCP2 protein. MeCP2 can be 443 subdivided into five domains corresponding to the $\mathrm{N}$-terminal domain (NTD), the methyl-CpG 444 binding domain (MBD), the intervening domain (ID), the transcriptional repression domain (TRD), 445 and the C-terminal domain (CTD). By analyzing the MECP2 mutation database, Bellini et al. 446 have reported that about $25 \%$ of residues in the CTD and ID have been associated with 447 pathogenic missense mutations in RTT ${ }^{38,73}$. In addition, the ID domain has been shown to be 448 involved in MeCP2-mediated multiple protein-protein interactions as well as diverse 449 phosphorylation events ${ }^{73}$. Interestingly, T203 is located within the ID domain (Fig. 1B). Therefore, 450 it is possible that the O-GIcNAcylation on T203 may affect its binding to DNA or its protein 451 partners, resulting in subsequent regulation of neurodevelopment. However, the precise 452 biological partners of T203 remain to be further identified. Our results showed that the T203M 453 mutation had no effect on its binding to histone deacetylase 1 (HDAC1) (Fig. S5), but did result 454 in reduced binding to the Bdnf IV promoter (Fig. 7). This suggests an HDAC1-independent 455 transcriptional activation mechanism may underlie T203 O-GlcNAcylation in cortical neurons.

456 Although increasing evidence points to O-GlcNAcylation as a mediator of 457 neurodegeneration and a modulator of neuronal signaling pathways in the brain $27,32,71,74,75,76$, $45877,78,79$, the underlying molecular mechanisms that support these crucial functions are not yet 459 fully understood. Previously, the O-GICNAcylation of CREB, a common upstream regulator of 460 Bdnf, was found to critically regulate neuronal gene expression, axonal and dendritic growth, 
and long-term memory ${ }^{76,79}$. MeCP2 associates with CREB1 at the promoter of an activated target but not a repressed target ${ }^{80}$. In addition, CREB signaling has also recently been shown to be involved in Rett syndrome pathogenesis ${ }^{81}$. Interestingly, hMeCP2 T203 O-GIcNAcylation, but not its T203M mutant was able to rescue the activity-dependent Bdnf transcription (Fig. 7B). Because T203M dramatically reduces O-GlcNAcylation of hMeCP2, it suggests that OGlcNAcyated T203 is necessary to support Bdnf transcription following neural depolarization. Interestingly, T203M did not notably impact Creb expression levels following $\mathrm{KCl}$ treatment (data not shown). This suggests that neuronal activation stimulates a variety of responses at both the O-GIcNAcylation PTM level and gene expression level to control neural development. First, both MeCP2 and CREB proteins are dynamically regulated via O-GIcNAcylation to stimulate normal dendrite and spine growth ${ }^{76}$. Second, neural activity may result in the increased association of the MeCP2 and CREB1 ${ }^{80}$, and the coupling between T203 O-GlcNAcylated MeCP2 with Bdnf exon IV, as well as the dissociation between phosphorylated MeCP2 with the Bdnf exon III promoter to promote Bdnf transcription ${ }^{21}$. Together, our data and previous results indicate that upon neuronal activation, O-GIcNAcylation of MeCP2 upregulates the CREB-BDNF signaling pathway via multiple mechanisms to promote neural development and synaptic plasticity, with T203 as a direct binding partner of the Bdnf promoter.

Given the prevalence of PTMs on MeCP2, it is interesting to further consider the role of OGlcNAc among other forms of PTM, especially in the context of RTT. Thus far, the best characterized PTM of MeCP2 protein is its phosphorylation ${ }^{21,22,23,24}$, and deregulation of MeCP2 phosphorylation may be involved in the pathogenesis of RTT ${ }^{65,66}$. Interestingly, O-GIcNAcylation has been closely linked to phosphorylation as both modifications can occur at the same or adjacent sites ${ }^{82}$, and functional interaction between both modifications has also been previously characterized ${ }^{83}$. However, in this study our MS assay did not identify the O-GIcNAc site T203 also as a phosphorylation site (Fig. 1 and Fig. S1), suggesting that the observed neurodevelopmental regulatory effects are likely due to O-GIcNAcylation on T203, rather than 
phosphorylation. In addition, we analyzed the effects of T203M deglycosylation on the phosphorylation of MeCP2 S421 and S80 22, 23, but did not find any significant changes in phosphorylation level on either site (Fig. S6). Thus, it is possible that T203 O-GlcNAcylation

490 affects neural development and activity-dependent transcription by independently recruiting a 491 specific set of molecular coactivator to target gene promoters. Given the complexity of various 492 genetic mutations in the pathogenesis of RTT ${ }^{38,84}$, and that some mutations impact PTM sites

$493{ }^{38}$, delineating the effects of disease-related PTM sites and their interaction may lead to novel 494 therapeutic targets for reversing neural deficiencies in RTT.

495 In this study, we identified several previously unknown O-GlcNAcylation sites in human 496 MeCP2 and revealed novel functions of T203 O-GlcNAc modification in the regulation of neural 497 development and synaptic transmission. Furthermore, we provided mechanistic insight into 498 downstream signaling of MeCP2 T203 O-GIcNAcylation, adding to our understanding of the 499 complex signaling network following MeCP2 PTMs in mediating neuronal activity-dependent 500 transcription. Future work will need to further map out major relevant molecular pathways 501 affected by MeCP2 T203 O-GIcNAcylation and their possible involvement in the pathogenesis 502 of RTT disorders. 


\section{Materials and Methods}

504

\section{Animals}

Pregnant ICR mice were purchased from SiBeiFu Co. (Beijing, China) and housed in the animal breeding facility of the Beijing Institute of Basic Medical Sciences. All animals were maintained and utilized in accordance with the guidelines of the Institutional Animal Care and Use Committee of the Beijing Institute of Basic Medical Sciences. MECP2 Tg mice (JAX lab, \#008679) ${ }^{19}$ were kindly provided by Dr. Zilong Qiu at the Institute of Neuroscience, Chinese Academy of Sciences. MECP2 Tg mice genotypes were determined by PCR assays using murine tail DNA, with primers as follows: 5'-CGCTCCGCCCTATCTCTGA-3' (forward) and 5'ACAGATCGGATAGAAGACTC-3' (reverse).

\section{Plasmids}

The rat Mecp2 gene expression plasmid was a gift from Dr. Zilong Qiu at the Institution of Neuroscience, Chinese Academy of Sciences. The human MECP2-e1 gene expression plasmid was a gift from Dr. Keping Hu at Chinese Academy of Medical Sciences \& Peking Union Medical College. N-terminal HA-tagged MECP2 and GFP-tagged MECP2 CDS were subcloned into the pXJ40-HA and pEGFP-C1 vector, respectively. Other constructs of various MECP2 mutations and indicated truncations were all generated based on the recombinant pXJ40-HA-hMeCP2 or pEGFP-hMeCP2 constructs. N-terminal His-tagged MECP2 and GST-tagged MECP2 CDS were subcloned into the pET-28a and pGEX-6P-1 vector, respectively. GST-tagged full length OGT was subcloned into the pET-28a plasmid. GST-OGT (323-1041) and GST-OGA (31-624) plasmids were gifts from Dr. Huadong Pei at National Center for Protein Sciences (Beijing). The PCR primers used for subcloning of truncated or mutant MeCP2 and OGT were listed in supplemental Table S3.

The MeCP2 LEMPRA (lentivirus-mediated protein-replacement assay) plasmid (named pLenti-FUGW-shMeCP2-GFP-IRES-Flag-MECP2) is a lentiviral vector with dual promoters. It 
was constructed by inserting the $\mathrm{H} 1$ promoter-driven mouse Mecp2-specific shRNA cassette against the sequence of 5'GTCAGAAGACCAGGATCTC-3' into the indicated site ${ }^{85}$, and inserting Flag-tagged shRNA-resistant human MECP2 coding sequence under the control of the Ubiquitin-C (Ubc) promoter. The shRNA-resistant Flag-MECP2 was generated by introducing five silent nucleotide mutations indicated in the following lower case letters within the coding sequence of MECP2: 5'- GagcGAAGACCAaGAcCTC-3' 24.

\section{Cell culture and DNA transfection}

HEK293T and Neuro2A cells were cultured in DMEM basic (Gibco, C11995500BT) and MEM basic (Gibco, C11095500BT) medium, respectively, supplemented with 10\% FBS (Gibco, 10099-141C), $100 \mathrm{U} / \mathrm{mL}$ penicillin-streptomycin (Gibco, 15140122), in a $37^{\circ} \mathrm{C}$ incubator with a humidified, 5\% CO $\mathrm{CO}_{2}$ atmosphere. Lipofectamine 2000 (Invitrogen, 11668019) was used for

\section{Mass spectrometry}

HA-MeCP2 was ectopically expressed in HEK293T cells. Proteins were isolated by co-IP, and eluted using $200 \mu \mathrm{g} / \mathrm{ml}$ of HA peptide. The endogenous mouse MeCP2 was enriched by co-

546 IP with anti-MeCP2 antibody (Cell Signaling Technology, 3456) and subjected to Sodium

547 dodecyl sulfate-polyacrylamide gel electrophoresis (SDS-PAGE). The gel was stained with

548 Coomassie brilliant blue. Visualized bands were excised, de-stained with ammonium

549 bicarbonate buffer, and dehydrated in $75 \%$ acetonitrile. Following rehydration (with $50 \mathrm{mM}$

550 ammonium bicarbonate), the gel slices were crushed and subjected to overnight digestion with

551 trypsin or chymotrypsin. The peptides were extracted with acetonitrile containing $0.1 \%$ formic

552 acid and vacuum dried. Proteolytic peptides were reconstituted with mobile phase A ( $2 \%$

553 acetonitrile containing $0.1 \%$ formic acid) and then separated on an on-line C18 column (75 $\mu \mathrm{m}$

554 inner diameter, $360 \mu \mathrm{m}$ outer diameter, $10 \mathrm{~cm}, 3 \mu \mathrm{m} \mathrm{C} 18)$. Mobile phase A consisted of $0.1 \%$ 
formic acid in $2 \%$ acetonitrile and mobile phase $B$ was $0.1 \%$ formic acid in $84 \%$ acetonitrile. A linear gradient from 3 to $100 \%$ B over 75 minutes at a flow rate of $350 \mathrm{~nL} / \mathrm{min}$ was applied. Mass spectrometry analysis was carried out on a Q-Exactive mass spectrometer (Thermo Fisher, SJ) operated in data dependent scan mode. Survey scan (m/z 375-1300) was performed at a resolution of 60,000 followed by MS2 scans to fragment the 50 most abundant precursors with collision induced dissociation. The activation time was set at $30 \mathrm{~ms}$, the isolation width was $1.5 \mathrm{amu}$, the normalized activation energy was $35 \%$, and the activation q was 0.25 . Mass spectrometry raw files were scanned and analyzed with the Proteome Discoverer software (version 2.1, Thermo Fisher Scientific) using MASCOT search engine with percolator against the human or rodent ref-sequence protein database. The mass tolerance was set to 20 ppm for precursor and 0.5 Da for product ion. Missed cleavages were no more than two for each peptide. O-GlcNAc of Ser/Thr were used as variable modifications.

\section{In vitro O-GIcNAcylation assay by chemoenzymatic labelling}

Chemoenzymatic labelling and biotinylation of proteins in cell lysates were carried out following the manufacturer's instructions. Briefly, WT and MECP2 Tg mice brain lysates $(200 \mu \mathrm{g})$ were labelled using the Click-iT O-GIcNAc Enzymatic Labelling System (Molecular Probes, C33368) protocol, then conjugated with an alkyne-biotin compound according to the Click-iT Protein Analysis Detection Kit (Molecular Probes, C33372) protocol. A parallel negative control experiment was performed in the absence of the labelling enzyme GalT or UDP-GalNAz. The methanol and chloroform were used to precipitate the biotinylated lysates. The biotinylated products were solubilized using $1 \%$ SDS solution, and neutralized with the neutralization buffer (6\% Nonidet P40, 100 mM Na $2 \mathrm{HPO}_{4}, 150 \mathrm{mM} \mathrm{NaCl}, 50 \mathrm{mM}$ Tris- $\mathrm{HCl}, \mathrm{pH}$ 7.5, and protease inhibitor cocktail). Lysates were then incubated with streptavidin resin with end-to-end rotation at $4^{\circ} \mathrm{C}$ overnight. Resin was then washed five times with low-salt buffer $\left(100 \mathrm{mM} \mathrm{Na}_{2} \mathrm{HPO}_{4}, 150 \mathrm{mM}\right.$ $\mathrm{NaCl}, 0.1 \%$ SDS, $1 \%$ Triton $\mathrm{X}-100,0.5 \%$ sodium deoxycholate) and five times with high-salt buffer 
(100 $\mathrm{mM} \mathrm{Na}_{2} \mathrm{HPO}_{4}, 500 \mathrm{mM} \mathrm{NaCl}, 0.2 \%$ Triton X-100). Biotinylated proteins were boiled with SDS anti-MeCP2 antibody (Cell Signaling Technology, 3456). To quantify the level of O-GIcNAcylation, the intensity of the total MeCP2 protein band (Input) and the O-GlcNAc MeCP2 protein band (Elution) were measured, and the ratio of the intensity of the O-GlcNAc protein versus the intensity of the total protein was taken as the level of O-GIcNAcylation ${ }^{86}$.

\section{Primary culture of cortical neurons}

Cortical neurons and hippocampal neurons were dissected and cultured from E15.5 and P1524). Neuronal cultures were treated overnight in $1 \mu \mathrm{M}$ tetrodotoxin (TTX) (Kangte Biotech, purity $>99 \% ; 121206$ ) to reduce endogenous neuronal activity prior to stimulation. Neurons were membrane depolarized with $55 \mathrm{mM}$ extracellular $\mathrm{KCl}$ as previously described ${ }^{21}$.

\section{Lentivirus package and purification}

Lentiviruses were produced by co-transfection of HEK293T cells with the MeCP2 LEMPRA plasmid and the helper plasmids psPAX2 and VSV-G. Lentiviruses were concentrated by ultra601 centrifugation $48-72 \mathrm{hr}$ after transfection, and viral titers were determined by infection of HEK293T cells and determined by qPCR. Primary cultured neurons were infected with the 603 purified lentivirus at appropriate time at $1 \times 10^{6} \mathrm{TU} / \mathrm{mL}$.

\section{Real time quantitative RT-PCR}

Total RNA was extracted using TRIzol (Invitrogen, 15596018). $0.5 \mu \mathrm{g}$ of RNA was used for reverse transcription using the Reverse Transcription System (TaKaRa, RR036) according to the 
608 manufacturer's protocol. Real-time PCR was conducted in triplicates employing SYBR Green 609 PCR master mix (CWBIO, CW2601) with the appropriate forward and reverse primers. For 610 quantitative analysis of gene expression, results were averaged from three replicates in three 611 independent experiments. Values were normalized to Actb levels. All PCR reactions were 612 performed in triplicate with the following primers. Bdnf total: 5'613 TGCCTAGATCAAATGGAGCTTCTC-3' (Forward) and 5'-CCGATATGTACTCCTGTTCTTCAGC-3' 614 (Reverse); Bdnf exon IV: 5'-CAGAGCAGCTGCCTTGATGTT-3' (Forward) and 5'615 GCCTTGTCCGTGGACGTTTA-3' (Reverse); Bdnf exon VI: 5'-GGGATCCGAGAGCTTTGTGTGGA$616 \quad$ 3' (Forward) and 5'-GTAGGCCAAGTTGCCTTGTCCGT-3' (Reverse); $\quad$ Acta2: 5'617 GAGCTACGAACTGCCTGACG-3' (Forward) and 5'-TACCCCCTGACAGGACGTTG-3' (Reverse); 618 Actb: 5'-GGCTGTATTCCCCTCCATCG-3' (Forward) and 5'-CCAGTTGGTAACAATGCCATGT-3' 619 (Reverse).

\section{Expression and purification of recombinant proteins}

Both His-tagged and GST-tagged proteins were expressed in Escherichia coli BL21.

623 Bacteria were treated with $0.1 \mathrm{mM}$ Isopropyl $\beta$-D-thiogalactoside (IPTG) (Thermo Fisher, AM9462) at $16^{\circ} \mathrm{C}$ for $16 \mathrm{hr}$ to induce protein expression. To purify the His-tagged recombinant protein, the induced bacteria were harvested and suspended in $10 \mathrm{mM} \mathrm{PBS}(\mathrm{pH} 7.4)$ containing $62620 \mathrm{mM}$ imidazole (Sigma-Aldrich, I2399) and $1 \mathrm{mM}$ Phenylmethanesulfonyl fluoride (PMSF) 627 (Sigma-Aldrich, 10837091001), followed by ultrasonication. The recombinant protein in the 628 supernatant was incubated with the Ni Magnetic beads for $2 \mathrm{hr}$ at $4^{\circ} \mathrm{C}$, washed in $10 \mathrm{mM}$ PBS 629 (pH 7.4) three times, eluted with 10 mM PBS ( $\mathrm{pH} 7.4)$ containing $200 \mathrm{mM}$ iminazole, and followed 630 by dialysis with $10 \mathrm{mM}$ PBS ( $\mathrm{pH}$ 7.4). Quantification of protein amount was measured by 631 Coomassie brilliant blue staining.

632 To purify GST-tagged recombinant proteins, induced bacteria were harvested and 633 suspended in $10 \mathrm{mM}$ PBS ( $\mathrm{pH}$ 7.4) containing $1 \mathrm{mM}$ PMSF, followed by ultrasonication. 
634 Recombinant GST-tagged protein in the supernatant was purified using glutathione-Sepharose 635 4B beads (GE Healthcare, 17-0756-01), washed in $10 \mathrm{mM}$ PBS (pH 7.4) three times, eluted with 63610 mM PBS (pH 7.4) containing 20 mM reduced glutathione, and followed by dialysis with 10 637 mM PBS ( $\mathrm{pH}$ 7.4). Quantification of the protein amount was measured by Coomassie brilliant 638 blue staining.

\section{In vitro O-GIcNAcylation assay}

Purified recombinant GST-OGT fusion protein (323-1041) was incubated with wild type His-

642 tagged recombinant hMeCP2 or various His-tagged hMeCP2 mutants in $50 \mu \mathrm{L}$ reactions (50 mM 643 Tris- $\mathrm{HCl}, 12.5 \mathrm{mM} \mathrm{MgCl}, 2 \mathrm{mM}$ UDP-GlcNAc $1 \mathrm{mM} \mathrm{DTT}, \mathrm{pH}$ 7.5) for overnight at $37^{\circ} \mathrm{C}$, and 644 Western blot analysis was carried out with anti-O-GlcNAc (RL2) antibody (Abcam, ab2739). For 645 O-GIcNAc cleavage assay, O-GlcNAcylated proteins were treated with purified recombinant 646 GST-OGA (31-624) fusion protein for $2 \mathrm{hr}$ at $37^{\circ} \mathrm{C}$ in a volume of $50 \mu \mathrm{L}$, and Western blot analysis 647 was carried out with anti-O-GIcNAc (RL2) antibody (Abcam, ab2739). Quantitative analysis of 648 recombinant protein was measured by Coomassie brilliant blue staining ${ }^{42}$.

\section{GST Pull-down assay}

Bacteria-expressed GST, GST-hMeCP2 or GST-OGT fusion proteins were immobilized on

652 glutathione Sepharose 4B beads (GE Healthcare, 17-0756-01) and washed three times with 653 GST binding buffer (50 mM Tris-HCl, pH 7.5, $100 \mathrm{mM} \mathrm{NaCl}, 50 \mathrm{mM} \mathrm{NaF}, 2 \mathrm{mM}$ EDTA, 1\% 654 Nonidet P40, and protease inhibitor cocktail). The beads were next incubated with His-hMeCP2 655 or His-OGT recombinant protein lysates at $4^{\circ} \mathrm{C}$ for $4 \mathrm{hr}$ under rotation. Beads were washed with 656 GST binding buffer (50 mM Tris-HCl, pH 7.5, $100 \mathrm{mM} \mathrm{NaCl}, 50 \mathrm{mM} \mathrm{NaF}, 2 \mathrm{mM}$ EDTA, 1\% 657 Nonidet P40, and protease inhibitor cocktail) and proteins were eluted, followed by Western blot 658 with indicated antibodies. 


\section{Co-immunoprecipitation (Co-IP) assay}

For endogenous co-IP assay, mouse brain lysates or cell lysates (1-2 mg) with protease inhibitor cocktail were incubated with anti-MeCP2 (Cell Signaling Technology, 3456) or anti-OGT (Sigma-Aldrich, HPA030751) antibody overnight at $4^{\circ} \mathrm{C}$. After incubation, protein-A/G agarose beads were used for precipitation for $2 \mathrm{hr}$. The precipitates were then washed 5 times with the lysis buffer and eluted by boiling in SDS sample buffer for Western blotting. The gel was transferred to PVDF membrane, and the membrane was blocked with $5 \%$ milk in TBST buffer for $1 \mathrm{hr}$ (at room temperature). It was then incubated overnight at $4^{\circ} \mathrm{C}$ with antibody, washed three times in TBST, and the signals were revealed by HRP reaction using the SuperSignal Chemilluminescent Substrate (Beyotime, P0018AS).

For co-IP in HEK293T cells, HEK293T cells were plated on $60 \mathrm{~mm}$ plates, and transfection was performed when the cells reached $50 \%$ confluence. Lipofectamine 2000 was used for transfection. A total of $5 \mu \mathrm{g}$ of DNA was used in $60 \mathrm{~mm}$ plates at a molar ratio of 1:1 for GFP. tagged and HA-tagged constructs. Cells were harvested $36 \mathrm{hr}$ later. The cells were rinsed with cold PBS, harvested, and lysed for $20 \mathrm{~min}$ at $4^{\circ} \mathrm{C}$ in a modified RIPA lysis buffer. $10 \%$ of the supernatant was saved for the input control, and the rest was incubated with $2 \mu \mathrm{g}$ anti-HA antibody (Cell Signaling Technology, 3724) or anti-GFP (Cell Signaling Technology, 2955) overnight at $4^{\circ} \mathrm{C}$. The immune complex was isolated by addition of $30 \mathrm{ml}$ of a $50 \%$ slurry of mixed protein-A/G agarose for $2 \mathrm{hr}$, washed three times with the lysis buffer, then eluted by boiling-SDS lysis, and resolved by $8 \%$ SDS-PAGE. The gel was transferred to PVDF membranes, and the membrane was blocked with $5 \%$ milk in TBST buffer for $1 \mathrm{hr}$ at room temperature. It was then incubated overnight at $4^{\circ} \mathrm{C}$ with the antibody, washed three times in TBST, and the signals were revealed by HRP reaction using the SuperSignal Chemiluminescent Substrate (Beyotime, P0018AS).

\section{Chromatin immunoprecipitation (ChIP) assay}


Chromatin immunoprecipitation (ChIP) was prepared using the Upstate Biotechnology and Abcam kit following the manufacturer's protocol. Briefly, Neuro2A cells were cross-linking at room temperature by addition of $1 \%$ formaldehyde for 15 min. Cross-linking was stopped by addition of $0.2 \mathrm{M}$ glycine for $5 \mathrm{~min}$ at room temperature. Cells were washed three times in $10 \mathrm{ml}$ ice-cold $10 \mathrm{mM}$ PBS $(\mathrm{pH} 7.4)$, re-suspended in lysis buffer $(50 \mathrm{mM}$ Tris- $\mathrm{HCl}$ at $\mathrm{pH} 8.0,1 \%$ SDS, $5 \mathrm{mM}$ EDTA, and protease inhibitors) and directly sheared by sonication and processed for ChIP assay. Lysates was precleared by incubating in $1 \mathrm{~mL}$ diluted chromatin with a salmon sperm DNA /protein A-Sepharose (50 $\mu \mathrm{L} 50 \%$ slurry in $10 \mathrm{mM}$ Tris- $\mathrm{HCl}, \mathrm{pH} 8.0,1 \mathrm{mM}$ EDTA) for $2 \mathrm{hr}$ at $4{ }^{\circ} \mathrm{C}$ with agitation. Beads were pelleted using brief centrifugation and the supernatant fraction collected. The rest of the supernatant was divided in two fractions: one for an equivalent amount of normal rabbit lgG control and the second incubated with ChIP-grade anti-MeCP2 (Abcam, ab2828) at $4^{\circ} \mathrm{C}$ with agitation overnight. Salmon sperm DNA/protein A-Sepharose slurry was added to the immune complexes and incubated at $4^{\circ} \mathrm{C}$ for $2-4 \mathrm{hr}$. Sepharose beads were collected and washed sequentially for 10 min each in the following buffers: once in low salt buffer (0.1\% SDS, 1\% Triton X-100, 2 mM EDTA, 20 mM Tris-HCl pH 8.0, $150 \mathrm{mM} \mathrm{NaCl}$ ), three times in high salt buffer $(0.1 \%$ SDS, 1\% Triton X-100, 2 mM EDTA, 20 mM Tris-HCl, pH 8.0, 500 mM $\mathrm{NaCl}$ ), twice in LiCl buffer (250 mM LiCl, 1\% Nonidet P40, 1\% deoxycholate, $10 \mathrm{mM}$ Tris-HCl pH

704 Complexes were eluted using elution buffer $(250 \mu \mathrm{l} \text { of } 1 \% \text { SDS and } 100 \mathrm{mM} \mathrm{NaHCO})_{3}$, 705 formaldehyde cross-links reversed and DNA was precipitated and re-suspended. Both input and 706 immunoprecipitated samples were analyzed by quantitative RT-PCR with Bdnf promotor IV

707 primers: 5'-GGTCTTTAAGGTGGCCCAAG-3' (forward) and
708 TGGAGCATGTGATCAAAACAA-3' (reverse).

\section{In utero electroporation}

711 In utero electroporation (IUE) of E13.5 pregnant mice (ICR; commercially obtained from 
712 SiBeiFu) was performed as previously described ${ }^{48}$. Specifically, pregnant mice were

713 anesthetized with isoflurane (Yuyanbio, Shanghai). The uterine horns were exposed by

714 Cesarean section and sterile, pre-warmed saline was repeatedly applied during the operation to

715 keep the intestines moist. Animals were kept on a heating pad during the entire operation. $1 \mu \mathrm{L}$

716 of the DNA plasmids for electroporation $(2 \mu \mathrm{g} / \mu \mathrm{L})$ was injected into the lateral ventricle through

717 a pulled glass capillary tube. DNA was electroporated into the neocortex. After electroporation,

718 the uterine horns were carefully repositioned into the abdominal cavity, which was then filled with

719 pre-warmed saline. Animals were left to recover in a clean cage and embryos allowed to continue

720 their development. At E17.5 or P15, pregnant mice or young mice were sacrificed. The brains

721 were dissected and post-fixed with $4 \%$ PFA overnight at $4^{\circ} \mathrm{C}$. Coronal brain slices from IUE mice

722 were prepared by Cryostat (Thermo Scientific, FSE) for immunofluorescent staining, or prepared

723 by the automated vibrating blade microtome (Leica, VT1200 S) for the electrophysiological

724 recording.

725

726

Immunofluorescent staining

The immunofluorescent staining of frozen brain sections and cultured neurons was

performed using standard techniques as previously described in our lab ${ }^{87,88}$. Briefly, frozen

729 sections $(30 \mu \mathrm{m})$ or cultured neurons were washed for 10 min with $0.5 \%$ Triton X-100/PBS

730 (PBS-T) for three times and then blocked with 5\% goat serum in PBST for $1 \mathrm{hr}$. The sections or

731 cultured neurons were then incubated overnight at $4^{\circ} \mathrm{C}$ with primary antibodies, washed for 10

732 min with $0.5 \%$ PBS-T for three times and subsequently treated with Alexa Fluor 568- or Alexa

733 Fluor 488-conjugated fluorescent secondary antibody (1:500; Biotium, 20103, 20012) $1 \mathrm{hr}$ at

734 room temperature. The nucleus was counterstained with DAPI (ZSGB-BIO, ZLI-9556) during

735 mounting onto glass slides with anti-fade solution. All images were processed and analyzed

736 using Olympus FV-1200 and Image J software. 


\section{Electrophysiology}

Preparation of brain slices was performed as previously described ${ }^{89,90}$. A single slice was then transferred to the recording chamber and submerged in a continuously flowing oxygenated

$741 \mathrm{NaHCO}_{3}$-buffered saline $(1.0-1.5 \mathrm{ml} / \mathrm{min})$ warmed at $32^{\circ} \mathrm{C}$. The recording electrodes had 742 resistance of 3-5 $\mathrm{M} \Omega$ when filled with internal solution consisting of (in $\mathrm{mM}$ ): $135 \mathrm{mM}$ cesium 743 methanesulfonate, $10 \mathrm{mM}$ Hepes, $0.2 \mathrm{mM}$ EGTA, $8 \mathrm{mM} \mathrm{NaCl}, 4 \mathrm{mM} \mathrm{Mg}-\mathrm{ATP}$, and $0.3 \mathrm{mM}$ $744 \mathrm{Na}_{3} \mathrm{GTP}(\mathrm{pH} 7.2$ with $\mathrm{CsOH}$, osmolality adjusted to $280-290 \mathrm{mOsm}$ ). The slice was visualized

745 with a $40 \times$ water-immersion objective (Olympus) using standard infrared and differential 746 interference contrast (IR-DIC) microscopy, and a CCD camera (QImaging, Surrey). Cells in the 747 cortex up to $\sim 60 \mu \mathrm{m}$ beneath the slice surface were patched and monitored. Recording in normal 748 voltage-clamp mode was performed with an Axon 700B amplifier (Molecular devices) and 749 Clampex 10.5 software (Molecular devices). After tight-seal (>1 G $\Omega$ ) formation, fast and slow 750 capacitance compensation was auto performed. Neurons were excluded from the analysis when 751 their series resistance was above $25 \mathrm{M} \Omega$ and changed by more than $25 \%$ during the experiment.

752 Data were filtered at $2 \mathrm{kHz}$ and acquired at a sampling rate of $10 \mathrm{kHz}$. For mEPSC recording, 753 the target neurons were held at $-70 \mathrm{mV}$ in the presence of SR95531 (25 $\mu \mathrm{M}$; Sigma-Aldrich, 754 S106) and TTX (1 $\mu \mathrm{M}$; Kangte Biotech, purity $>99 \%$; 121206). For mIPSC recording, the target neurons were held at $0 \mathrm{mV}$ in the presence of CNQX (25 $\mu \mathrm{M}$; Sigma-Aldrich, C239), DL-AP5 (50 $\mu \mathrm{M}$; Abcam, ab120271), and TTX (1 $\mu \mathrm{M}$, Kangte Biotech, purity > 99\%; 121206). 5 min consecutive miniature events were collected and analyzed. All mEPSCs or mIPSCs above a threshold value (5 pA) were included in the data analysis and each event was verified visually. Experiments were carried out in a genotype-blinded manner. No statistical analysis was used to predetermine the sample sizes used for experiments; however, our sample sizes are similar to 761 those reported previously.

762 All drugs were purchased from Sigma-Aldrich (St. Louis, MI, USA) unless otherwise noted.

763 Drugs were dissolved as concentrated stocks and stored at $-20^{\circ} \mathrm{C}$. Working solutions with 
764 different drugs were prepared just before use. During experiments, drugs were applied in the

765 flowing bath solutions. Total replacement of the medium in the recording chamber occurred within

$7661 \mathrm{~min}$. Data analysis was performed with software including Clampfit (Version 10.5), MiniAnalysis

767 program (Version 6.0.3) Prism (Version 8.0), and Origin (Version 9.0).

768

769

\section{Dendritic length and spine density analysis}

770

The length of dendritic branches in primary cultured hippocampus neurons was determined as follows: EGFP-positive neurons were randomly selected from each condition, and the dendritic length of all protrusions was analyzed using Fiji software. At least three independent experiments were performed, and the number of neurons $>50$ per condition were analyzed. For spine density analysis, confocal Z stacks of neurons in hippocampal were acquired with a confocal microscope (Olympus, FV-1200) using an oil-immersion 60 X objective lens. Images were analyzed with Fiji software. Protrusions in direct contact with the dendrites were counted as spines, and the average spine density was calculated as the number of spines per $\mu \mathrm{m}$ dendritic length. At least $500 \mu \mathrm{m}$ dendrites from seven or more neurons were analyzed for each groups (more than 3 mice per group). All quantifications were analyzed with One-way analysis of variance (ANOVA).

\section{Statistical analysis}

Data are presented as mean \pm SEM and were analyzed by two-tailed Student's t-test, or oneway ANOVA followed by Bonferroni test. Kruskal-Wallis ANOVA followed by Dunn's post-hoc test was performed to analyze the data of electrophysiological experiments. A $\chi^{2}$ test was applied to analyze the distribution of cells in either layers of the neocortex, and the distribution of different types of dendritic spines. Unless otherwise indicated, in figures, ${ }^{*} p<0.05$, ${ }^{* *} p<0.01$, and ${ }^{* * *} p<$ 


\section{SUPPLEMENTAL INFORMATION}

791 Supplemental Information includes three tables and six figures can be found with this article as a 792 separate supplementary file.

794 AUTHOR CONTRIBUTIONS

795 H.W. designed the experiments. X.D. performed the MS experiment to identify the O-

796 GlcNAcylation sites on MeCP2. J.C., L.C., R.D. and Y.W. performed the lentivirus packaging and 797 purification, biochemical and molecular biology experiments. J.C. and Q.Z. performed the in utero 798 electroporation experiments. Z.Z. and J.C. performed the electrophysiology experiments. H.W., 799 X.D., M.F., J.C. and Z.Z. analyzed the results. H.W. and J.C. wrote the manuscript.

\section{ACKNOWLEDGMENTS}

802 We thank Dr. Zilong Qiu at Institute of Neuroscience, CAS, Dr. Huadong Pei at the National Center 803 for Protein Sciences (Beijing), and Dr. Ke-Ping Hu at Chinese Academy of Medical Sciences \& 804 Peking Union Medical College for providing the valuable reagents for this work, and thank Dr. 805 Dong Yang at Beijing Proteome Research Center for his great help with the phylogeny tree analysis of MeCP2 protein sequence. We thank all members of the Wu laboratory for discussion.

807 This work was supported by the National Natural Science Foundation of China (Grants 31770929

808 and 31522029), and the Beijing Municipal Science and Technology Commission (Grants 809 Z181100001518001 and Z161100000216154) to H.W.

810

\section{CONFLICT OF INTEREST}

812 The authors declare that they have no conflicts of interest with the contents of this article. 
817 (A) The phylogeny tree of MeCP2 protein sequence from 25 representative species. The length of each branch represents the evolutionary distance between the MeCP2 protein sequences. The dashed scale line indicates the values of evolutionary distance.

820 (B) MS identified O-GlcNAcylation sites in rodent and human MeCP2 protein. hMeCP2, human 821 MeCP2; rMeCP2, rat MeCP2; mMeCP2, mice MeCP2; NTD, the N-terminal domain; MBD, the 822 methyl-CpG binding domain; ID, the intervening domain; TRD, the transcriptional repression 823 domain; CTD, the C-terminal domain.

824 (C-F) Four representative mass spectra of O-GlcNAcylation sites in hMeCP2 are shown. hMeCP2 was purified from HEK293T cells and analyzed by MS to identify the O-GlcNAcylation sites.

(G) Multiple-sequence alignment of MeCP2 protein to reveal the conservation of O-GlcNAcylation site. The O-GIcNAcylation sites in MeCP2 from eight different representative species are shown. The identified O-GIcNAcylation sites are shown in red, and T203 site is highlighted in yellow.

\section{Figure 2. OGT Directly Interacts with and O-GIcNAcylates MeCP2}

831 (A) The mouse brain lysates were immunoprecipitated with an anti-MeCP2 antibody, followed by

832 Western blot analysis with an anti-OGT antibody.

833 (B) Reciprocal co-IP assay of mouse brain lysates with an anti-OGT antibody, followed by Western 834 blot analysis with an anti-MeCP2 antibody.

835 (C-D) GST pull-down assays for His-hMeCP2 and GST-OGT, or His-OGT and GST-hMeCP2. 836 Input and pull-down samples were analyzed with anti-MeCP2 and anti-OGT antibodies. Input 837 represents $5 \%$ of the amount used for pull-down assay.

838 (E-F) Cell lysates from HEK293T cells transfected with HA-tagged hMeCP2 and GFP-tagged full length and indicated deletion mutants of OGT were immunoprecipitated with anti-HA or anti-GFP 
antibodies, followed by Western blot analysis with anti-GFP and anti-HA antibodies, respectively.

841 Input represents $5 \%$ of the amount used for pull-down assay.

842 (G-H) Representative quantification of Western blot results followed by co-IP assay in $E$ and $F$, 843 respectively. Histograms show mean \pm SEM. One-way ANOVA followed by Bonferroni test, ${ }^{* *} p<$ $844 \quad 0.01$.

845 (I) A summarized diagram for the serial deletion mutants of OGT and their binding capacities to 846 MeCP2 is shown. Deletion of either the entire TPRs or 1-3 TPRs domain within OGT dramatically 847 disrupts its binding to MeCP2.

\section{Figure 3. T203 Residue of hMeCP2 is Dynamically O-GIcNAcylated by OGT}

850 (A) A schematic depicting the chemoenzymatic labelling approach for biotinylation, capture, and detection of O-GIcNAcylated protein from brain or cell lysates.

(B) Detection of O-GlcNAcylated MeCP2 protein in brain lysates from wild type (WT) and MECP2 transgenic (Tg) mice using chemoenzymatic labelling approach. Higher level of O-GlcNAcylated MeCP2 was detected in MECP2 Tg mice compared with WT control. In the absence of GalT or UDP-GalNAz, no O-GlcNAcylated MeCP2 can be detected. HSP70 was used as loading control. co-expressed with or without HA-tagged OGT in HEK293T cells for co-IP assay followed by

858 Western blot analysis with anti-MeCP2 and anti-RL2 antibodies, respectively. Input represents 5\% 859 of the amount used for co-IP assay. pEGFP-C1 mock vector was used as a negative control.

860 (D) OGT directly O-GlcNAcylates MeCP2 by in vitro glycosylation assay. Recombinant His-tagged 861 hMeCP2 protein was incubated with or without purified GST-OGT (323-1041) and UDP-GIcNAc, 862 followed by Western blot analysis with an anti-RL2 antibody. The loading amount of recombinant 863 proteins used for the in vitro assay was confirmed by Coomassie blue staining.

864 (E-F) Identification of the O-GlcNAc modified sites in MeCP2 by in vitro glycosylation assay. 865 Purified wild type or mutant His-hMeCP2 was used as substrates of GST-OGT (323-1041) in the 
866

867

868

869

870

871

872

873

874

875

876

877

878

879

880

881

882

883

884

885

886

887

888

889

890

891

presence of UDP-GICNAc. The loading amount of GST-OGT and His-hMeCP2 was confirmed by Coomassie blue staining. Quantification analysis shows the significantly decreased O-GIcNAc level in the hMeCP2 mutations including T203M, S204A, T436A and 4Muts. Histograms show mean \pm SEM. One-way ANOVA followed by Bonferroni test, ${ }^{* * *} p<0.001$.

(G-H) Co-IP assay shows dramatically reduced the O-GlcNAc modified level in T203M or 4Muts of hMeCP2 compared with WT in the presence of OGT in transfected HEK293T cells. Cell lysates were immunoprecipitated with anti-GFP beads and immunoblotted as indicated in G. Input represents $5 \%$ of the total amount used for co-IP assay. pEGFP-C1 mock vector was used as a negative control. Quantification analysis shows the significantly decreased RL2 level in T203M and 4Muts mutants compared with wild type control. Histograms show mean \pm SEM. One-way ANOVA followed by Bonferroni test, ${ }^{* * *} p<0.001$.

(I-J) OGA reversely regulates the O-GlcNAcylation of hMeCP2 by in vitro glycosylation assay. Quantification analysis shows the significantly decreased RL2 level of hMeCP2 in the presence of GST-OGA. Histograms show mean \pm SEM. $t$-test, ${ }^{* * *} p<0.001$.

(K-L) The O-GlcNAcylation of T203M mutant or 4Muts mutant of hMeCP2 was also reversed by OGA by in vitro glycosylation assay. Quantification analysis shows the significantly decreased RL2 level of WT and hMeCP2 mutants in the presence of GST-OGA. Histograms show mean \pm SEM. One-way ANOVA followed by Bonferroni test, ${ }^{* * *} p<0.001$.

\section{Figure 4. T203 O-GIcNAcylation is Required for Dendritic Spine Formation and Soma Size}

\section{Maintenance in Cultured Hippocampal Neurons}

(A) The recombinant lentivirus-mediated protein-replacement assay construct, pLEMPRAMeCP2, was generated to knockdown endogenous mMeCP2 and express Flag-tagged ectopic $\mathrm{hMeCP} 2$.

(B) Schematic of the experimental design. The primary hippocampal neurons were isolated from E17.5 mouse embryos for in vitro culture. The neurons at DIV 7 were infected with the indicated 
LEMPRA lentivirus for 7 days, and then fixed at DIV 14 for immunofluorescent staining and confocal imaging.

894 (C) Representative pictures of mouse primary hippocampal neurons infected with indicated lentivirus at DIV 7, lentivirus FUGW-GFP was used as negative control. Neurons were collected and fixed at DIV 14 for immunofluorescent staining with anti-PSD-95 antibody for measurement of dendritic spines. The soma and dendrites of indicated LEMPRA lentivirus infected positive

898 neurons were illustrated by GFP (green), and the distribution of PSD-95 positive dendritic spines

899 were shown in red. Boxed areas of PSD-95 positive puncta (red) along the secondary dendritic 900 branches are shown at higher magnification to illustrate the detailed dendritic spine density (Zoom 901 panels). Scale bar represents $10 \mu \mathrm{m}$ and $5 \mu \mathrm{m}$, respectively.

902 (D) Quantification of the area of soma of indicated lentivirus infected positive neurons. Histograms 903 show mean \pm SEM. One-way ANOVA followed by Bonferroni test, ${ }^{* *} p<0.01$.

904 (E-F) Quantification of the linear density and the area of PSD-95 puncta along the secondary 905 dendritic branches in indicated lentivirus infected positive neurons, respectively. Histograms show mean \pm SEM. One-way ANOVA followed by Bonferroni test, ${ }^{* *} p<0.01,{ }^{* * *} p<0.001$.

\section{Figure 5. T203 O-GIcNAcylation is Required for Dendritic Spine Morphogenesis in vivo}

909 (A) Schematic of the experimental design. The indicated plasmids were in utero electroporated in

910 the neocortex of E13.5 mouse embryos. The electroporated region was then isolated at E17.5 or

911 P15 for neuronal migration assay or dendritic spine morphogenesis assay, respectively.

912 (B) Distribution of GFP+ pyramidal neurons in the indicated plasmid electroporated neocortex at

913 E17.5. Boxed areas of GFP+ neurons are shown at higher magnification to illustrate the detailed 914 distribution within the neocortex. The neocortex was equally divided into three parts from the 915 inside out showing as the inner layer, the intermediate layer, and the outer layer (Zoom panels). 916 The representative single GFP+ neuron from indicated groups was traced and illustrated by Fiji 917 software (Trace panels). Scale bars represents $200 \mu \mathrm{m}, 100 \mu \mathrm{m}$, and $25 \mu \mathrm{m}$, respectively. 
918 (C) Quantification of the relative ratio of GFP+ neuron distribution (\%) in distinct neocortical layers.

919 Histograms show mean \pm SEM. $\chi^{2}$-test, ${ }^{* *} p<0.01,{ }^{* * *} p<0.001$

920 (D) Quantification of the length of leading process (LP) in GFP+ neurons electroporated with the

921 indicated plasmid. Histograms show mean \pm SEM. One-way ANOVA followed by Bonferroni test, $922 * * * p<0.001$

923 (E) Representative images of dendritic spines on apical or basal dendrites of GFP+ neurons at

924 P15 after electroporation with the indicated plasmid. Boxed areas of basal and apical dendritic

925 fragments are shown at higher magnification to illustrate the detailed dendritic spine morphology

926 (Zoom panels). Scale bar represents $100 \mu \mathrm{m}, 50 \mu \mathrm{m}$, and $3 \mu \mathrm{m}$, respectively.

927 (F-G) Quantification of the dendritic spine density on apical and basal dendrites in GFP+ neurons, 928 respectively. Histograms show mean \pm SEM. One-way ANOVA followed by Bonferroni test, ${ }^{*} p<$ $9290.05,{ }^{* *} p<0.01,{ }^{* * *} p<0.001$.

$930(\mathrm{H})$ Quantification of the distribution of three subtypes of dendritic spines in GFP+ neurons at P15. 931 Histograms show mean \pm SEM. $\chi^{2}$-test, ${ }^{*} p<0.05,{ }^{* *} p<0.01,{ }^{* * *} p<0.001$

Figure 6. T203 O-GIcNAcylation is Essential for the Excitatory Synaptic Transmission in the Neocortex

935 (A) Schematic of the experimental design. The indicated plasmids were in utero electroporated in

936 the neocortex of E13.5 mouse embryos. The electroporated region was then isolated at P21, and 937 coronal brain slices were prepared for Patch clamp recording.

938 (B) A representative GFP+ recording cell electroporated with indicated plasmid showing in A.

939 (C) Representative traces of mEPSC recorded in Layer II/III GFP+ neurons electroporated with 940 indicated plasmids.

941 (D-E) Quantification of the amplitude and cumulative distributions of mEPSC in recorded GFP+ 942 neurons electroporated with indicated plasmid. Histograms show mean \pm SEM. Kruskal-Wallis 943 ANOVA followed by Dunn's post-hoc test, $p>0.05$ for all comparisons. 
944 (F-G) Quantification of the frequency and cumulative distributions of mEPSC in recorded GFP+

945 neurons electroporated with indicated plasmid. Histograms show mean \pm SEM. Kruskal-Wallis

946 ANOVA followed by Dunn's post-hoc test, ${ }^{*} p<0.05,{ }^{* *} p<0.01$, n.s., not significant.

$947(\mathrm{H})$ Representative traces of mIPSC recorded in Layer II/III GFP+ neurons electroporated with 948 indicated plasmid.

949 (I-J) Quantification of the amplitude and cumulative distributions of mIPSC in recorded GFP+ 950 neurons electroporated with indicated plasmid. Histograms show mean \pm SEM. Kruskal-Wallis 951 ANOVA followed by Dunn's post-hoc test. No significant differences were detected among each 952 group, $p>0.9999$ for all comparisons.

953 (K-L) Quantification of the frequency and cumulative distributions of mIPSC in recorded GFP+ 954 neurons electroporated with indicated plasmid. Histograms show mean \pm SEM. Kruskal-Wallis ANOVA followed by Dunn's post-hoc test. No significant differences were detected among each group, $p>0.9999$ for all comparisons. mouse embryos for in vitro culture. The neurons at DIV 3 were infected with the indicated lentivirus.

961 The final concentration of $1 \mu \mathrm{M}$ TTX was added in the culture medium at DIV 7 . After $12 \mathrm{hr}$, the 962 cultured cortical neurons were treated with $55 \mathrm{mM} \mathrm{KCl}$ for $5 \mathrm{hr}$ to trigger synchronous membrane 963 depolarization. Then the cultured neurons were harvested for RNA extraction and Q-PCR analysis.

964 (B-E) Quantification of the transcription of total Bdnf, Bdnf exon IV, Bdnf exon VI, and Acta2 at 965 mRNA level in response to $\mathrm{KCl}$ treatment. Histograms show mean \pm SEM. One-way ANOVA 966 followed by Bonferroni test, ${ }^{*} p<0.05,{ }^{* * *} p<0.001$.

967 (F) Schematic of the experimental design. The cultured Neuro2A cells were transfected with the 968 indicated plasmids for rescue experiments, and $48 \mathrm{hr}$ later, the transfected cells were collected 969 for ChIP assay. 
970 (G) A schematic depicting the total nine exons and exon IV promoter within Bdnf locus.

$971(\mathrm{H})$ Quantification of the ChIP assay. The binding activity of Bdnf exon IV promoter to MeCP2 in

972 indicated transfected cells were measured by Q-PCR amplification. Histograms show mean \pm

973 SEM. One-way ANOVA followed by Bonferroni test, ${ }^{* *} \mathrm{p}<0.01$. 


\section{REFERENCES}

1. Laurvick CL, Msall ME, Silburn S, Bower C, Klerk Nd, Leonard H. Physical and Mental Health of Mothers Caring for a Child With Rett Syndrome. Pediatrics 2006, 118(4): e1152-e1164.

2. Zoghbi Huda Y. Rett Syndrome and the Ongoing Legacy of Close Clinical Observation. Cell 2016, 167(2): 293-297.

3. Amir RE, Van dV, Ignatia B., Wan M, Tran CQ, Francke U, Zoghbi HY. Rett syndrome is caused by mutations in X-linked MECP2, encoding methyl-CpG-binding protein 2. Nature Genetics 1999, 23(2): 185-188.

4. Neul JL, Fang P, Barrish J, Lane J, Caeg EB, Smith EO, et al. Specific mutations in Methyl-CpGBinding Protein 2 confer different severity in Rett syndrome. Neurology 2008, 70(16): 1313-1321.

5. T. B. MECP2 mutations account for most cases of typical forms of Rett syndrome. Human Molecular Genetics 2000, 9(9): 1377-1384.

6. Ariani F, Mari F, Pescucci C, Longo I, Bruttini M, Meloni I, et al. Real-time quantitative PCR as a routine method for screening large rearrangements in Rett syndrome: Report of one case of MECP2 deletion and one case of MECP2 duplication. Human Mutation 2004, 24(2): 172-177.

7. Esch HV, Bauters M, Ignatius J, Jansen M, Froyen G. Duplication of the MECP2 Region Is a Frequent Cause of Severe Mental Retardation and Progressive Neurological Symptoms in Males. American Journal of Human Genetics 2005, 77(3): 442-453.

8. Meins. Submicroscopic duplication in Xq28 causes increased expression of the MECP2 gene in a boy with severe mental retardation and features of Rett syndrome. Jmedgenet 2005, 42(2): e12-e12.

9. Chen RZ, Akbarian S, Tudor M, Jaenisch R. Deficiency of methyl-CpG binding protein-2 in CNS neurons results in a Rett-like phenotype in mice. Nature Genetics 2001, 27(3): 327-331.

10. Tate P, Skarnes W, Bird A. The methyl-CpG binding protein MeCP2 is essential for embryonic development in the mouse. Nature Genetics 1996, 12(2): 205-208.

11. Marchetto MC, Carromeu C, Acab A, Yu D, Yeo GW, Mu Y, et al. A model for neural development and treatment of Rett syndrome using human induced pluripotent stem cells. Cell 2010, 143(4): 527-539.

12.Li Y, Wang H, Muffat J, Cheng AW, Orlando DA, Lovén J, et al. Global Transcriptional and 
1013 Translational Repression in Human-Embryonic-Stem-Cell-Derived Rett Syndrome Neurons. Cell Stem 1014 Cell 2013, 13(4): 446-458.

1015

1016

1017

1018

1019

1020

1021

1022

1023

1024

1025

1026

1027

1028

1029

1030

1031

1032

1033

1034

1035

1036

1037

1038

1039

1040

1041

1042

1043

1044

1045

1046

1047

1048

1049

1050

1051

13. Chapleau CA, Calfa GD, Lane MC, Albertson AJ, Larimore JL, Kudo S, et al. Dendritic spine pathologies in hippocampal pyramidal neurons from Rett syndrome brain and after expression of Rettassociated MECP2 mutations. Neurobiology of Disease 2009, 35(2): 219-233.

14. Schüle B, Armstrong DD, Vogel H, Oviedo A, Francke U. Severe congenital encephalopathy caused by MECP2 null mutations in males: central hypoxia and reduced neuronal dendritic structure. Clinical Genetics 2008, 74.

15.Kishi N, Macklis JD. MECP2 is progressively expressed in post-migratory neurons and is involved in neuronal maturation rather than cell fate decisions. Molecular \& Cellular Neuroscience 2004, 27(3): 306-321.

16. Guy J, Gan J, Selfridge J, Cobb S, Bird A. Reversal of Neurological Defects in a Mouse Model of Rett Syndrome. Science 2007, 315(5815): 1143-1147.

17.P. M. Learning and Memory and Synaptic Plasticity Are Impaired in a Mouse Model of Rett Syndrome. Journal of Neuroscience 2006, 26(1): 319-327.

18. Na ES, Nelson ED, Kavalali ET, Monteggia LM. The impact of MeCP2 loss- or gain-of-function on synaptic plasticity. Neuropsychopharmacology 2013, 38(1): 212-219.

19. Collins AL, Levenson JM, Vilaythong AP, Richman R, Armstrong DL, Noebels JL, et al. Mild overexpression of MeCP2 causes a progressive neurological disorder in mice. Hum Mol Genet 2004, 13(21): 2679-2689.

20.Liu Z, Li X, Zhang J-T, Cai Y-J, Cheng T-L, Cheng C, et al. Autism-like behaviours and germline transmission in transgenic monkeys overexpressing MeCP2. Nature 2016, 530(7588): 98.

21.Chen WG, Chang Q, Lin Y, Meissner A, West AE, Griffith EC, et al. Derepression of BDNF transcription involves calcium-dependent phosphorylation of MeCP2. Science 2003, 302(5646): 885-889.

22. Martinowich K, Hattori D, Wu H, Fouse S, He F, Hu Y, et al. DNA methylation-related chromatin remodeling in activity-dependent BDNF gene regulation. Science 2003, 302(5646): 890-893.

23. Tao J, Hu K, Chang Q, Wu H, Sherman NE, Martinowich K, et al. Phosphorylation of MeCP2 at Serine 80 regulates its chromatin association and neurological function. Proceedings of the National 
Academy of Sciences 2009, 106(12): 4882-4887.

24.Zhou Z, Hong EJ, Cohen S, Zhao W-n, Ho H-yH, Schmidt L, et al. Brain-specific phosphorylation of $\mathrm{MeCP} 2$ regulates activity-dependent Bdnf transcription, dendritic growth, and spine maturation. Neuron 2006, 52(2): 255-269.

25.Rutlin M, Nelson SB. MeCP2: phosphorylated locally, acting globally. Neuron 2011, 72(1): 3-5.

26. Olivier-Van Stichelen S, Wang P, Comly M, Love DC, Hanover JA. Nutrient-driven O-linked Nacetylglucosamine (O-GlcNAc) cycling impacts neurodevelopmental timing and metabolism. $J$ Biol Chem 2017, 292(15): 6076-6085.

27.Lagerlof O, Hart GW, Huganir RL. O-GlcNAc transferase regulates excitatory synapse maturity. Proc Natl Acad Sci U S A 2017, 114(7): 1684-1689.

28. Hwang H, Rhim H. Acutely elevated O-GlcNAcylation suppresses hippocampal activity by modulating both intrinsic and synaptic excitability factors. Sci Rep 2019, 9(1): 7287.

29. Hart GW. Three Decades of Research on O-GlcNAcylation - A Major Nutrient Sensor That Regulates Signaling, Transcription and Cellular Metabolism. Front Endocrinol (Lausanne) 2014, 5: 183.

30.Bond MR, Hanover JA. A little sugar goes a long way: the cell biology of O-GlcNAc. J Cell Biol 2015, 208(7): 869-880.

31.Hart GW, Housley MP, Slawson C. Cycling of O-linked $\beta$-N-acetylglucosamine on nucleocytoplasmic proteins. Nature 2007, 446(7139): 1017-1022.

32. Rexach JE, Clark PM, Hsieh-Wilson LC. Chemical approaches to understanding O-GlcNAc glycosylation in the brain. Nature Chemical Biology 2008, 4(2): 97-106.

33. Parween S, Varghese DS, Ardah MT, Prabakaran AD, Mensah-Brown E, Emerald BS, et al. Higher O-GlcNAc Levels Are Associated with Defects in Progenitor Proliferation and Premature Neuronal Differentiation during in-Vitro Human Embryonic Cortical Neurogenesis. Front Cell Neurosci 2017, 11: 415.

34. Wheatley EG, Albarran E, White CW, 3rd, Bieri G, Sanchez-Diaz C, Pratt K, et al. Neuronal OGlcNAcylation Improves Cognitive Function in the Aged Mouse Brain. Curr Biol 2019, 29(20): 33593369 e3354. 
35. Wang Z, Udeshi ND, O'Malley M, Shabanowitz J, Hunt DF, Hart GW. Enrichment and site mapping of O-linked $\mathrm{N}$-acetylglucosamine by a combination of chemical/enzymatic tagging, photochemical cleavage, and electron transfer dissociation mass spectrometry. Mol Cell Proteomics 2010, 9(1): 153-160.

36. Rexach JE, Rogers CJ, Yu S-H, Tao J, Sun YE, Hsieh-Wilson LC. Quantification of Oglycosylation stoichiometry and dynamics using resolvable mass tags. Nature Chemical Biology 2010, 6(9): 645-651.

37.Krishnaraj R, Ho G, Christodoulou J. RettBASE: Rett Syndrome Database Update. Human Mutation 2017, 00: 1-10.

38. Bellini E, Pavesi G, Barbiero I, Bergo A, Chandola C, Nawaz MS, et al. MeCP2 post-translational modifications: a mechanism to control its involvement in synaptic plasticity and homeostasis? Front Cell Neurosci 2014, 8: 236.

39. Kharrat M, Triki C, Maalej M, Ncir S, Ammar M, Kammoun F, et al. First description of an unusual novel double mutation in MECP2 co-occurring with the m.827A $>$ G mutation in the MT-RNR1 gene associated with angelman-like syndrome. Int J Dev Neurosci 2019, 79: 37-44.

40.Lombardi LM, Baker SA, Zoghbi HY. MECP2 disorders: from the clinic to mice and back. The Journal of clinical investigation 2015, 125(8): 2914-2923.

41. Thompson JW, Griffin ME, Hsieh-Wilson LC. Methods for the detection, study, and dynamic profiling of O-GlcNAc glycosylation. Methods in enzymology, vol. 598. Elsevier, 2018, pp 101-135.

42.Peng C, Zhu Y, Zhang W, Liao Q, Chen Y, Zhao X, et al. Regulation of the Hippo-YAP pathway by glucose sensor O-GlcNAcylation. Molecular cell 2017, 68(3): 591-604. e595.

43. Cheng TL, Wang Z, Liao Q, Zhu Y, Zhou WH, Xu W, et al. MeCP2 suppresses nuclear microRNA processing and dendritic growth by regulating the DGCR8/Drosha complex. Dev Cell 2014, 28(5): 547560.

44. Ramocki MB, Peters SU, Tavyev YJ, Zhang F, Carvalho CMB, Schaaf CP, et al. Autism and other neuropsychiatric symptoms are prevalent in individuals with $\mathrm{MeCP} 2$ duplication syndrome. Annals of Neurology 2009, 66(6): 771-782.

45.Jiang M, Ash RT, Baker SA, Suter B, Ferguson A, Park J, et al. Dendritic arborization and spine dynamics are abnormal in the mouse model of MECP2 duplication syndrome. J Neurosci 2013, 33(50): 
19518-19533.

46. Wood L, Gray NW, Zhou Z, Greenberg ME, Shepherd GM. Synaptic circuit abnormalities of motor-frontal layer 2/3 pyramidal neurons in an RNA interference model of methyl-CpG-binding protein 2 deficiency. J Neurosci 2009, 29(40): 12440-12448.

47. Chao HT, Zoghbi HY, Rosenmund C. MeCP2 controls excitatory synaptic strength by regulating glutamatergic synapse number. Neuron 2007, 56(1): 58-65.

49. Yanagida M, Miyoshi R, Toyokuni R, Zhu Y, Murakami F. Dynamics of the leading process, nucleus, and Golgi apparatus of migrating cortical interneurons in living mouse embryos. Proc Natl Acad Sci U S A 2012, 109(41): 16737-16742. developing and adult hippocampal pyramidal neurons using focal in utero electroporation. J Neurosci 2007 , 27(19): 5007-5011.

50. Belichenko PV, Oldfors A, Hagberg B, Dahlstrom A. Rett syndrome: 3-D confocal microscopy of cortical pyramidal dendrites and afferents. Neuroreport 1994, 5(12): 1509-1513.

51.Sala C, Segal M. Dendritic spines: the locus of structural and functional plasticity. Physiol Rev 2014, 94(1): 141-188.

52.Peters A, Kaiserman-Abramof IR. The small pyramidal neuron of the rat cerebral cortex. The synapses upon dendritic spines. Z Zellforsch Mikrosk Anat 1969, 100(4): 487-506.

53. Neul JL, Zoghbi HY. Rett syndrome: a prototypical neurodevelopmental disorder. Neuroscientist 2004, 10(2): 118-128.

54.Blackman MP, Djukic B, Nelson SB, Turrigiano GG. A critical and cell-autonomous role for MeCP2 in synaptic scaling up. $J$ Neurosci 2012, 32(39): 13529-13536.

55. Qiu Z, Sylwestrak EL, Lieberman DN, Zhang Y, Liu XY, Ghosh A. The Rett syndrome protein MeCP2 regulates synaptic scaling. J Neurosci 2012, 32(3): 989-994.

56. Dani VS, Nelson SB. Intact long-term potentiation but reduced connectivity between neocortical layer 5 pyramidal neurons in a mouse model of Rett syndrome. J Neurosci 2009, 29(36): 11263-11270.

57.Lonetti G, Angelucci A, Morando L, Boggio EM, Giustetto M, Pizzorusso T. Early environmental 
enrichment moderates the behavioral and synaptic phenotype of MeCP2 null mice. Biol Psychiatry 2010, 67(7): 657-665.

$$
\text { 58.Poo MM. Neurotrophins as synaptic modulators. Nat Rev Neurosci 2001, 2(1): 24-32. }
$$

59. Tanaka J, Horiike Y, Matsuzaki M, Miyazaki T, Ellis-Davies GC, Kasai H. Protein synthesis and neurotrophin-dependent structural plasticity of single dendritic spines. Science 2008, 319(5870): 16831687.

60.Figurov A, Pozzo-Miller LD, Olafsson P, Wang T, Lu B. Regulation of synaptic responses to highfrequency stimulation and LTP by neurotrophins in the hippocampus. Nature 1996, 381(6584): 706-709.

61.Luine V, Frankfurt M. Interactions between estradiol, BDNF and dendritic spines in promoting memory. Neuroscience 2013, 239: 34-45. in calcium regulation of BDNF expression. Neuron 1998, 20(4): 727-740.

63. Tao X, West AE, Chen WG, Corfas G, Greenberg ME. A calcium-responsive transcription factor, CaRF, that regulates neuronal activity-dependent expression of BDNF. Neuron 2002, 33(3): 383-395.

64. Tai DJC, Liu YC, Hsu WL, Ma YL, Cheng SJ, Liu SY, et al. MeCP2 SUMOylation rescues Mecp2-mutant-induced behavioural deficits in a mouse model of Rett syndrome. Nature Communications 2016, 7(1): 10552 .

65. Cohen S, Gabel Harrison W, Hemberg M, Hutchinson Ashley N, Sadacca LA, Ebert Daniel H, et al. Genome-Wide Activity-Dependent MeCP2 Phosphorylation Regulates Nervous System Development and Function. Neuron 2011, 72(1): 72-85.

66. Ebert DH, Gabel HW, Robinson ND, Kastan NR, Hu LS, Cohen S, et al. Activity-dependent phosphorylation of MeCP2 threonine 308 regulates interaction with NCoR. Nature 2013, 499(7458): 341345.

67.Pandey S, Simmons GE, Jr., Malyarchuk S, Calhoun TN, Pruitt K. A novel MeCP2 acetylation site regulates interaction with ATRX and HDAC1. Genes \& cancer 2015, 6(9-10): 408-421.

68. Choudhary C, Kumar C, Gnad F, Nielsen ML, Rehman M, Walther TC, et al. Lysine acetylation targets protein complexes and co-regulates major cellular functions. Science 2009, 325(5942): 834-840. 
69. Lefebvre T, Guinez C, Dehennaut V, Beseme-Dekeyser O, Morelle W, Michalski J-C. Does OGlcNAc play a role in neurodegenerative diseases? Expert Review of Proteomics 2005, 2(2): 265-275.

70.Lefebvre T, Caillet-Boudin ML, Buee L, Delacourte A, Michalski JC. O-GlcNAc glycosylation and neurological disorders. Advances in Experimental Medicine and Biology 2003, 535: 189-202.

71.Liu F, Iqbal K, Grundke-Iqbal I, Hart GW, Gong CX. O-GlcNAcylation regulates phosphorylation of tau: a mechanism involved in Alzheimer's disease. Proc Natl Acad Sci U S A 2004, 101(29): 1080410809.

72.Lagerlöf O, Hart GW, Huganir RL. O-GlcNAc transferase regulates excitatory synapse maturity. Proceedings of the National Academy of Sciences 2017, 114(7): 1684-1689.

73.Bedogni F, Rossi RL, Galli F, Cobolli Gigli C, Gandaglia A, Kilstrup-Nielsen C, et al. Rett syndrome and the urge of novel approaches to study MeCP2 functions and mechanisms of action. Neurosci Biobehav Rev 2014, 46 Pt 2: 187-201.

74. Khidekel N, Ficarro SB, Peters EC, Hsieh-Wilson LC. Exploring the O-GlcNAc proteome: direct identification of O-GlcNAc-modified proteins from the brain. Proc Natl Acad Sci U S A 2004, 101(36): 13132-13137.

75. Tallent MK, Varghis N, Skorobogatko Y, Hernandez-Cuebas L, Whelan K, Vocadlo DJ, et al. In vivo modulation of O-GlcNAc levels regulates hippocampal synaptic plasticity through interplay with phosphorylation. J Biol Chem 2009, 284(1): 174-181.

76. Rexach JE, Clark PM, Mason DE, Neve RL, Peters EC, Hsieh-Wilson LC. Dynamic O-GlcNAc modification regulates CREB-mediated gene expression and memory formation. Nat Chem Biol 2012, 8(3): 253-261.

77. Griffith LS, Mathes M, Schmitz B. $\beta$-Amyloid precursor protein is modified with O-linked Nacetylglucosamine. Journal of neuroscience research 1995, 41(2): 370-278.

78. Khidekel N, Ficarro SB, Clark PM, Bryan MC, Swaney DL, Rexach JE, et al. Probing the dynamics of O-GlcNAc glycosylation in the brain using quantitative proteomics. Nature Chemical Biology 2007, 3(6): 339-348.

79.Lamarre-Vincent N, Hsieh-Wilson LC. Dynamic Glycosylation of the Transcription Factor CREB: A Potential Role in Gene Regulation. Journal of the American Chemical Society 2003, 125(22): 66126613. 
80. Chahrour M, Jung SY, Shaw C, Zhou X, Wong ST, Qin J, et al. MeCP2, a key contributor to neurological disease, activates and represses transcription. Science 2008, 320(5880): 1224-1229.

81.Bu Q, Wang A, Hamzah H, Waldman A, Jiang K, Dong Q, et al. CREB Signaling Is Involved in

82. Hart GW, Slawson C, Ramirez-Correa G, Lagerlof O. Cross talk between O-GlcNAcylation and phosphorylation: roles in signaling, transcription, and chronic disease. Annu Rev Biochem 2011, 80: 825858.

83.Hu P, Shimoji S, Hart GW. Site-specific interplay between O-GlcNAcylation and phosphorylation in cellular regulation. FEBS Lett 2010, 584(12): 2526-2538.

1272

84. Shah RR, Bird AP. MeCP2 mutations: progress towards understanding and treating Rett syndrome. Genome Med 2017, 9(1): 17.

85.Lois C, Hong EJ, Pease S, Brown EJ, Baltimore D. Germline Transmission and Tissue-Specific Expression of Transgenes Delivered by Lentiviral Vectors. Science 2002, 295(5556): 868-872.

86. Rao X, Duan X, Mao W, Li X, Li Z, Li Q, et al. O-GlcNAcylation of G6PD promotes the pentose phosphate pathway and tumor growth. Nat Commun 2015, 6: 8468.

87.Wu H, Barik A, Lu Y, Shen C, Bowman A, Li L, et al. Slit2 as a beta-catenin/Ctnnb1-dependent retrograde signal for presynaptic differentiation. Elife 2015, 4.

88. Yang H, Zhu Q, Cheng J, Wu Y, Fan M, Zhang J, et al. Opposite regulation of Wnt/beta-catenin and Shh signaling pathways by Rack1 controls mammalian cerebellar development. Proc Natl Acad Sci U S A 2019, 116(10): 4661-4670.

1283

89. Yang H, Yang C, Zhu Q, Wei M, Li Y, Cheng J, et al. Rack1 Controls Parallel Fiber-Purkinje Cell Synaptogenesis and Synaptic Transmission. Front Cell Neurosci 2019, 13: 539.

90.Zhao Z, Zhang K, Liu X, Yan H, Ma X, Zhang S, et al. Involvement of HCN Channel in Muscarinic Inhibitory Action on Tonic Firing of Dorsolateral Striatal Cholinergic Interneurons. Front Cell Neurosci 2016, 10: 71. 
bioRxiv preprint doi: https://doi.org/10.1101/2020.06.17.158311; this version posted June 18, 2020. The copyright holder for this preprint (which was not certified by peer review) is the author/funder. All rights reserved. No reuse allowed without permission.

A

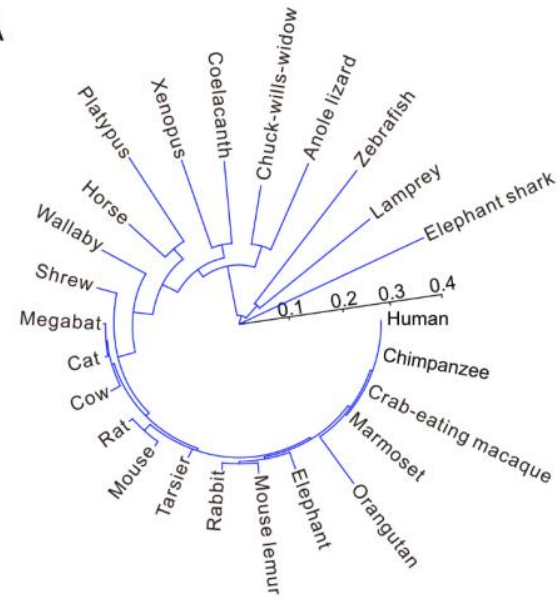

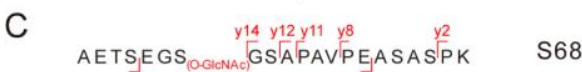

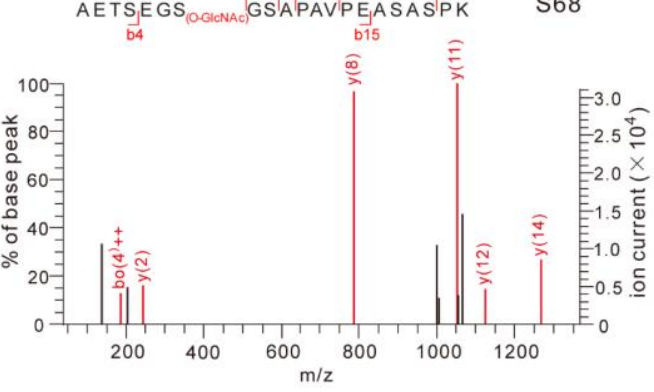

$\mathrm{E}$
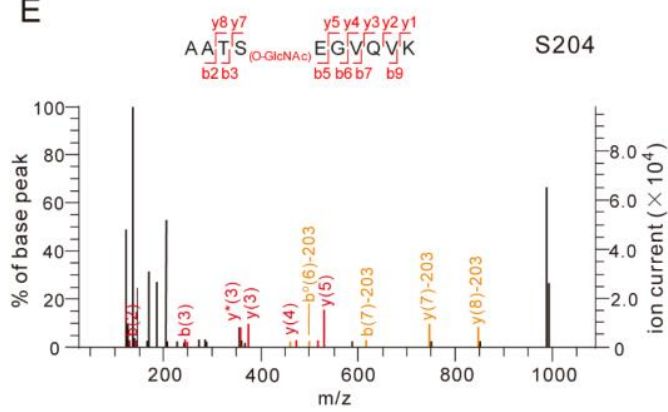
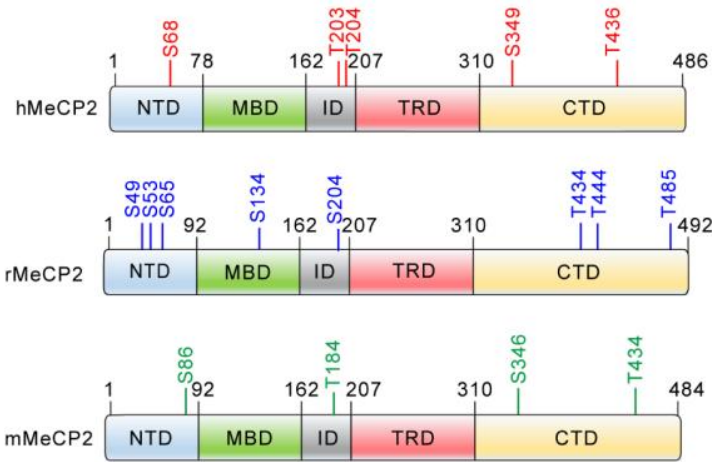

D

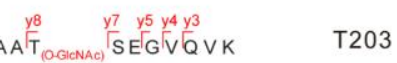

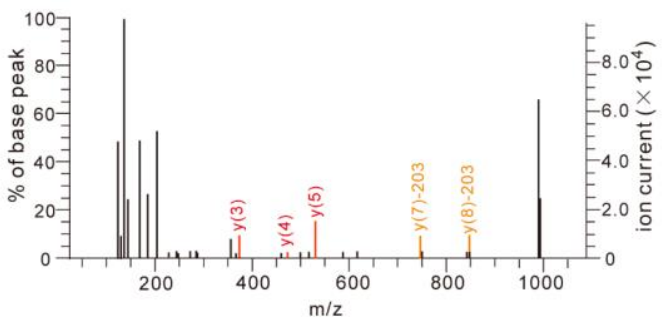

$\mathrm{F}$
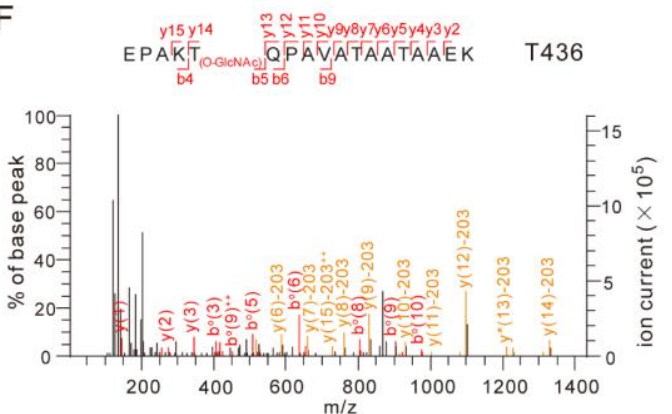

G

\section{守}

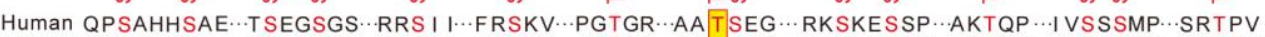

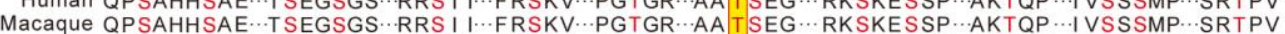

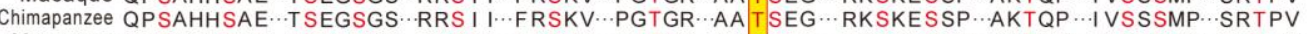

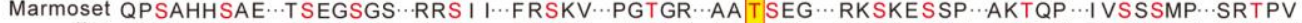

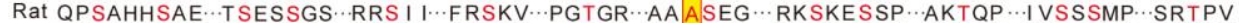

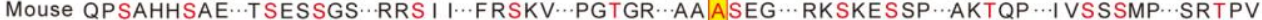

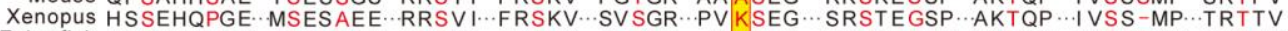

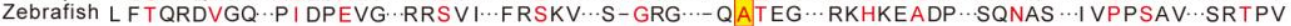

Figure 1. Identification of O-GIcNAcylation Sites in Rodent and Human MeCP2 
bioRxiv preprint doi: https://doi.org/10.1101/2020.06.17.158311; this version posted June 18, 2020. The copyright holder for this preprint (which was not certified by peer review) is the author/funder. All rights reserved. No reuse allowed without permission.

A

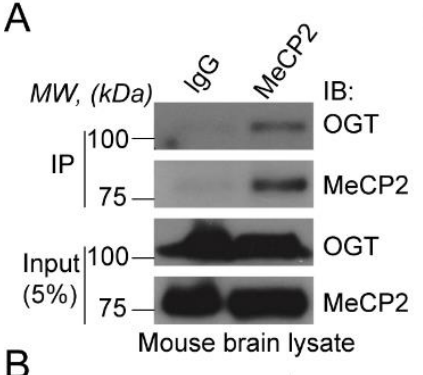

$M W,(k D a)$ के $0^{\circ} \quad$ IB:

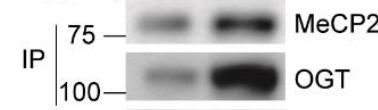

Input $75-\infty$ MeCP2

(5\%) 100 OGT

Mouse brain lysate
C

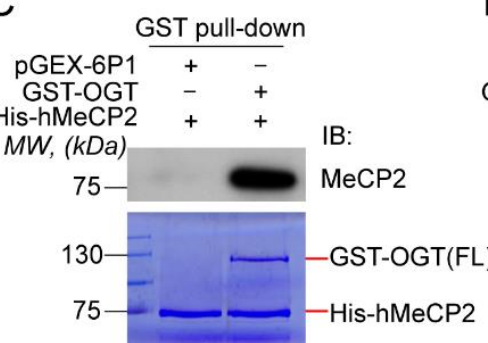

D

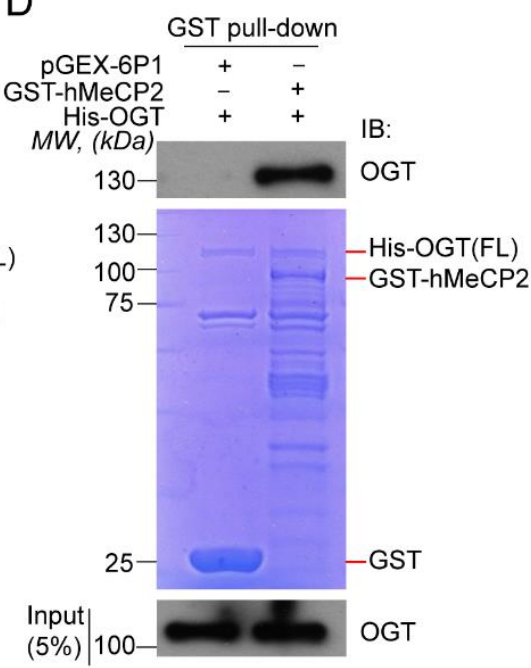

E
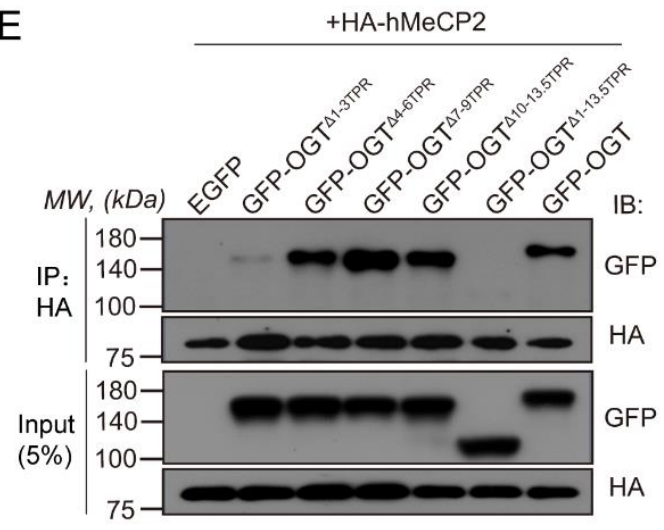

+HA-hMeCP2

$\mathrm{F}$

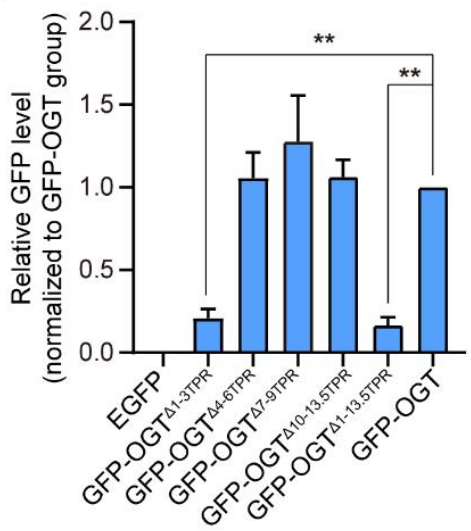

$\mathrm{H}$

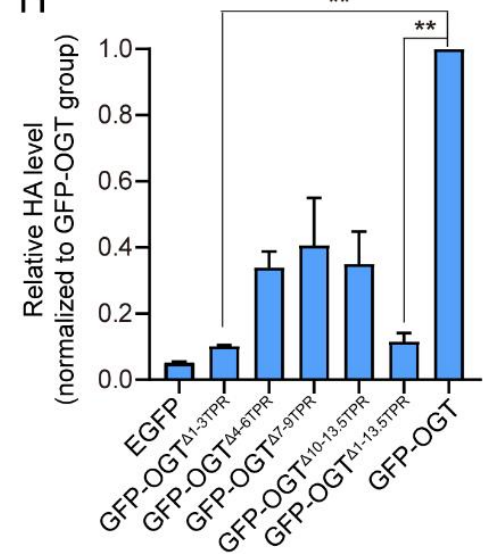

$\mathrm{I} \mathrm{MeCP} 2-+++-+$ binding

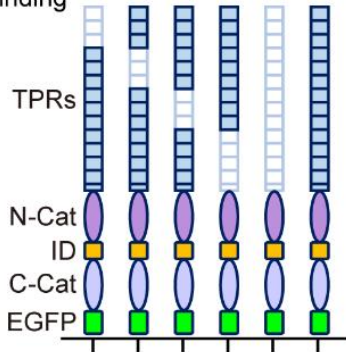

Figure 2. OGT Directly Interacts with and O-GIcNAcylates MeCP2 
bioRxiv preprint doi: https://doi.org/10.1101/2020.06.17.158311; this version posted June 18, 2020. The copyright holder for this preprint (which was not certified by peer review) is the author/funder. All rights reserved. No reuse allowed without permission.

A

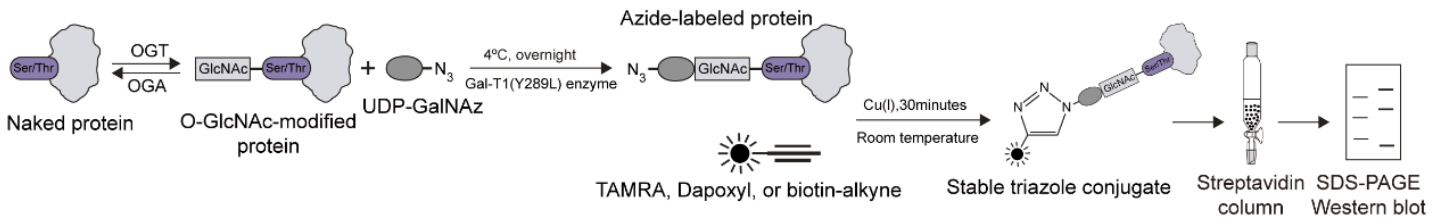

B

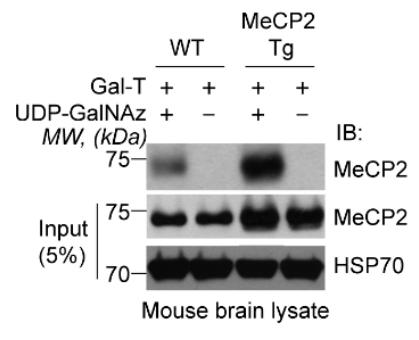

C

pEGFP-C1 + + - -

GFP-hMeCP2 --++
HA-OGT -+-+

IP. $\mid 100-$ RL2

GFP $100-\quad \mathrm{MeCP} 2$

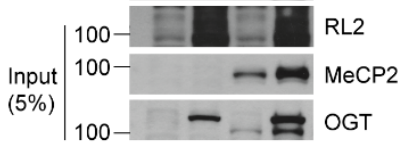

D

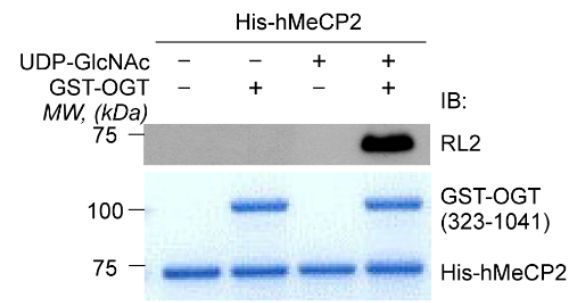

E

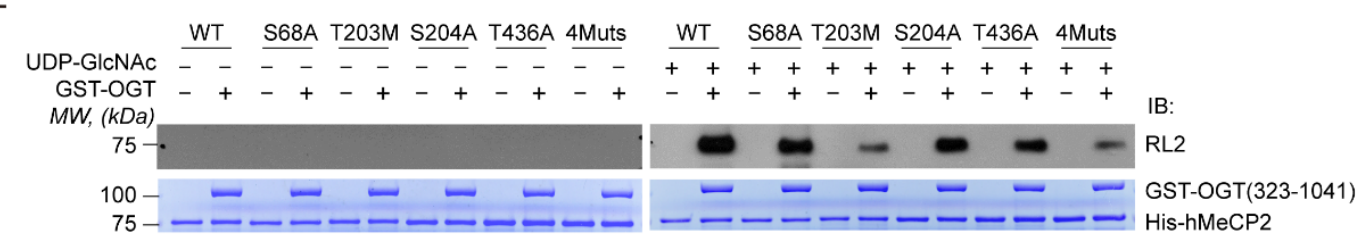

$\mathrm{F}$

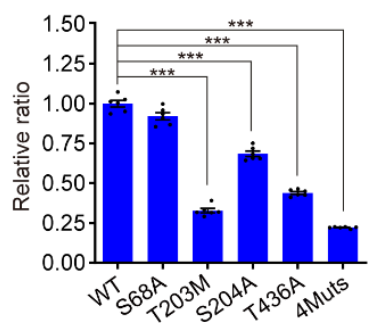

I

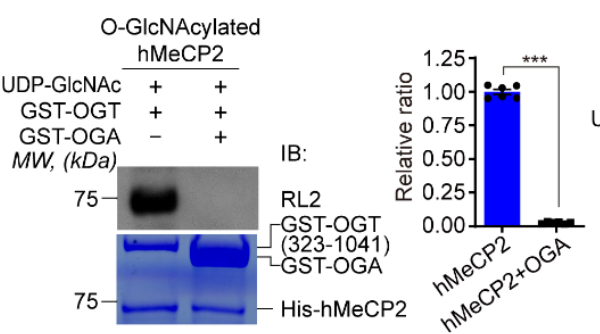

G

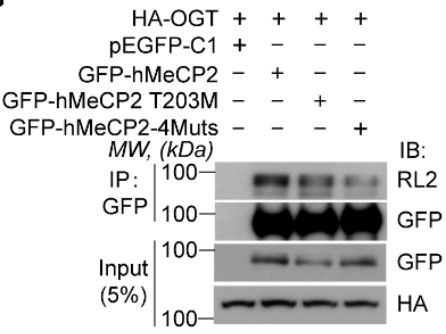

$\mathrm{K}$

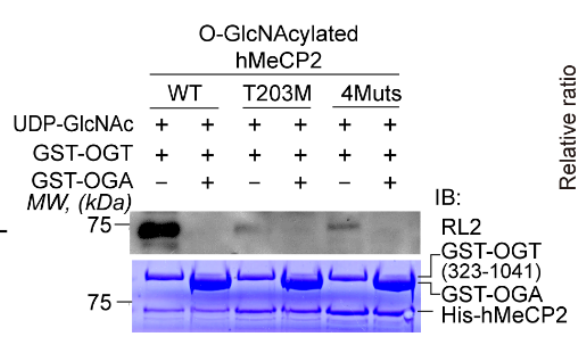

$\mathrm{H}$

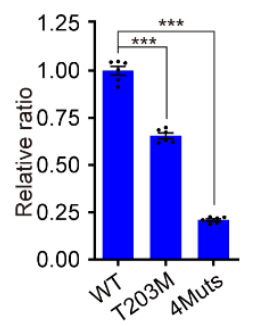

$\mathrm{L}$

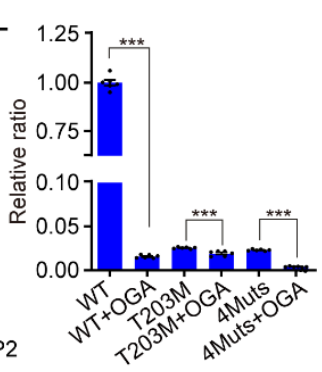

Figure 3. T203 Residue of hMeCP2 is Dynamically O-GIcNAcylated by OGT 
bioRxiv preprint doi: https://doi.org/10.1101/2020.06.17.158311; this version posted June 18, 2020. The copyright holder for this preprint (which was not certified by peer review) is the author/funder. All rights reserved. No reuse allowed without permission.
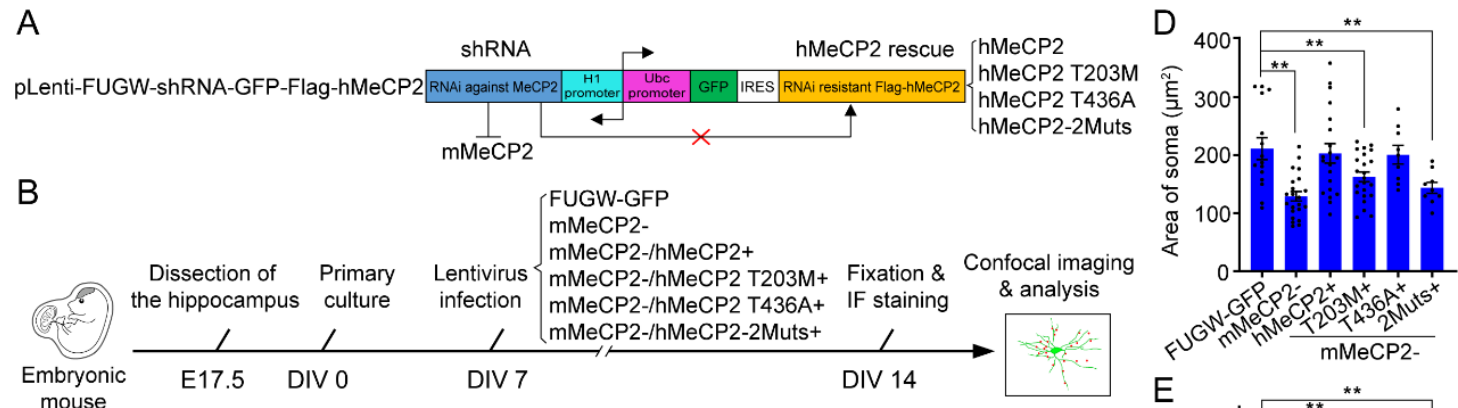

mouse

DIV 7

DIV 14

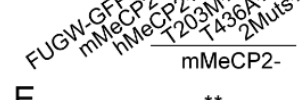

C
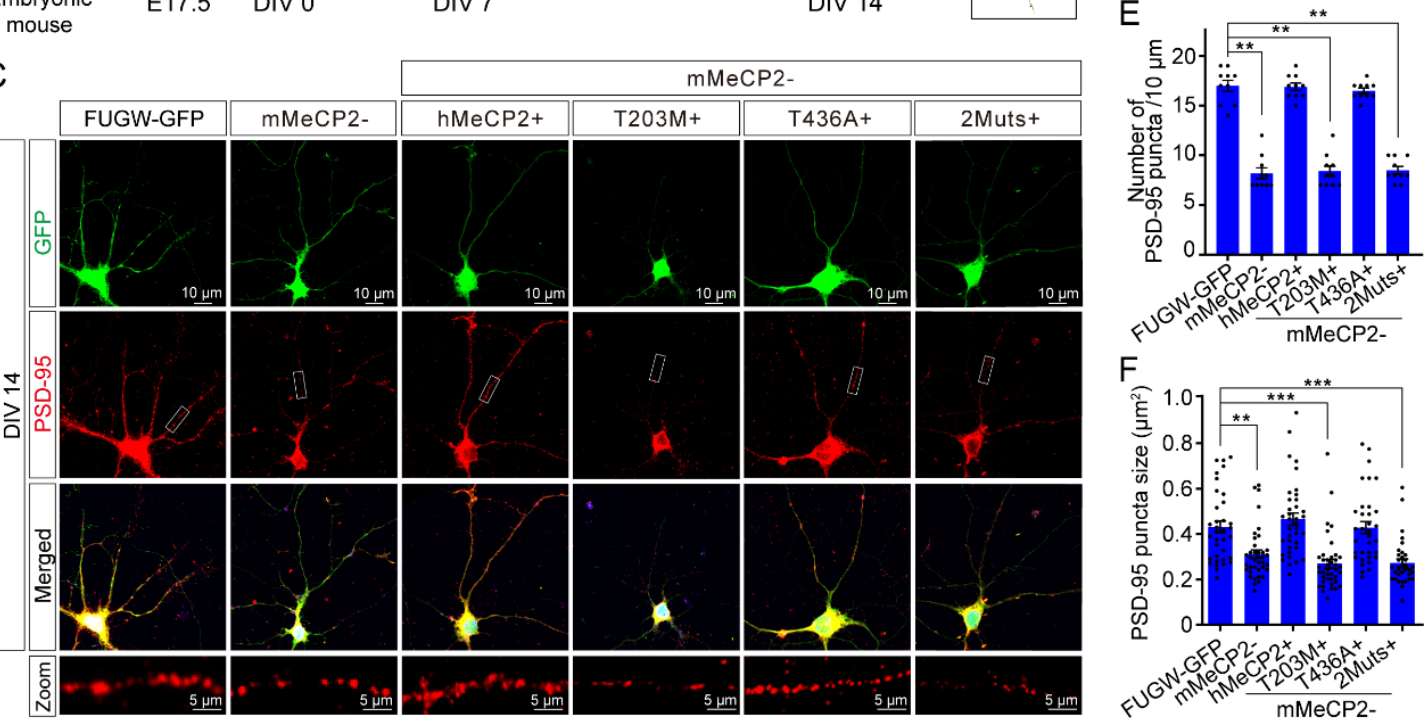

Figure 4. T203 O-GIcNAcylation is Required for Dendritic Spine Formation and Soma Size Maintenance in Cultured Hippocampal Neurons 
bioRxiv preprint doi: https://doi.org/10.1101/2020.06.17.158311; this version posted June 18, 2020. The copyright holder for this preprint (which was not certified by peer review) is the author/funder. All rights reserved. No reuse allowed without permission.

A

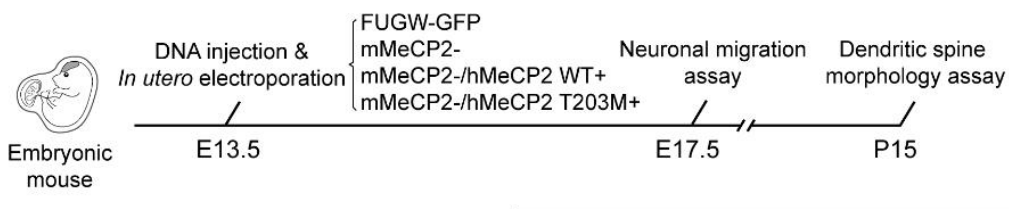

B

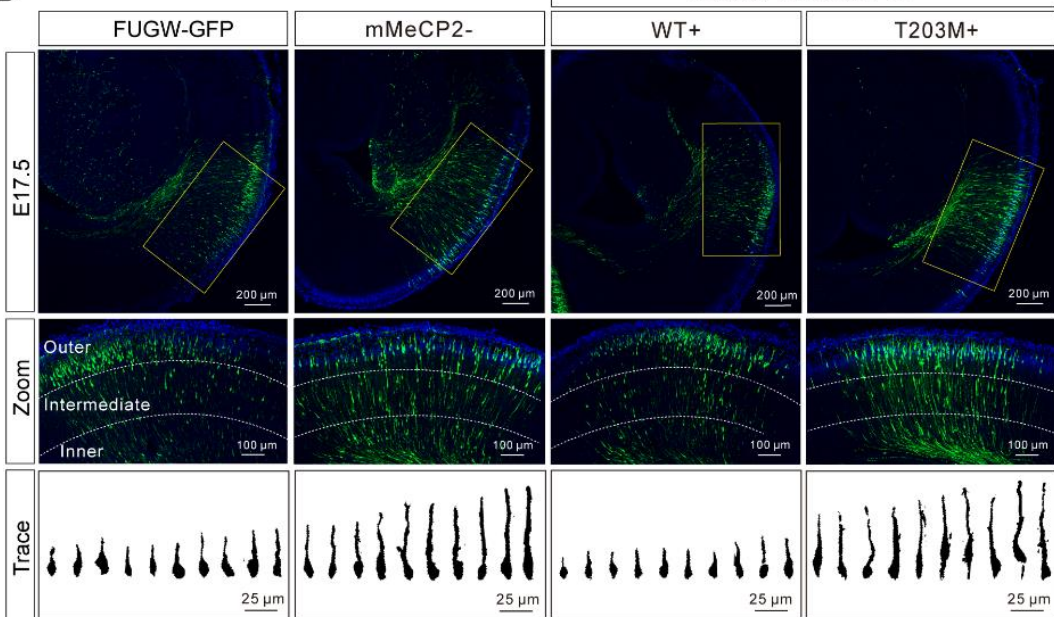

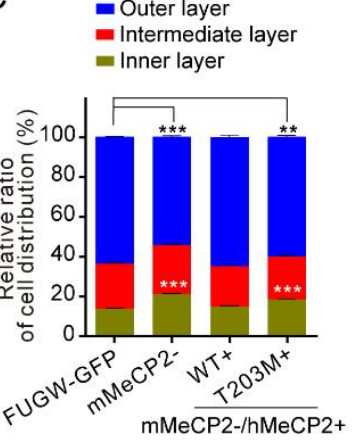

D

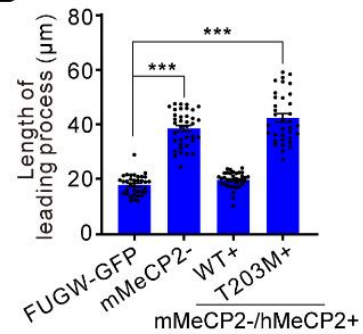

$\mathrm{E}$
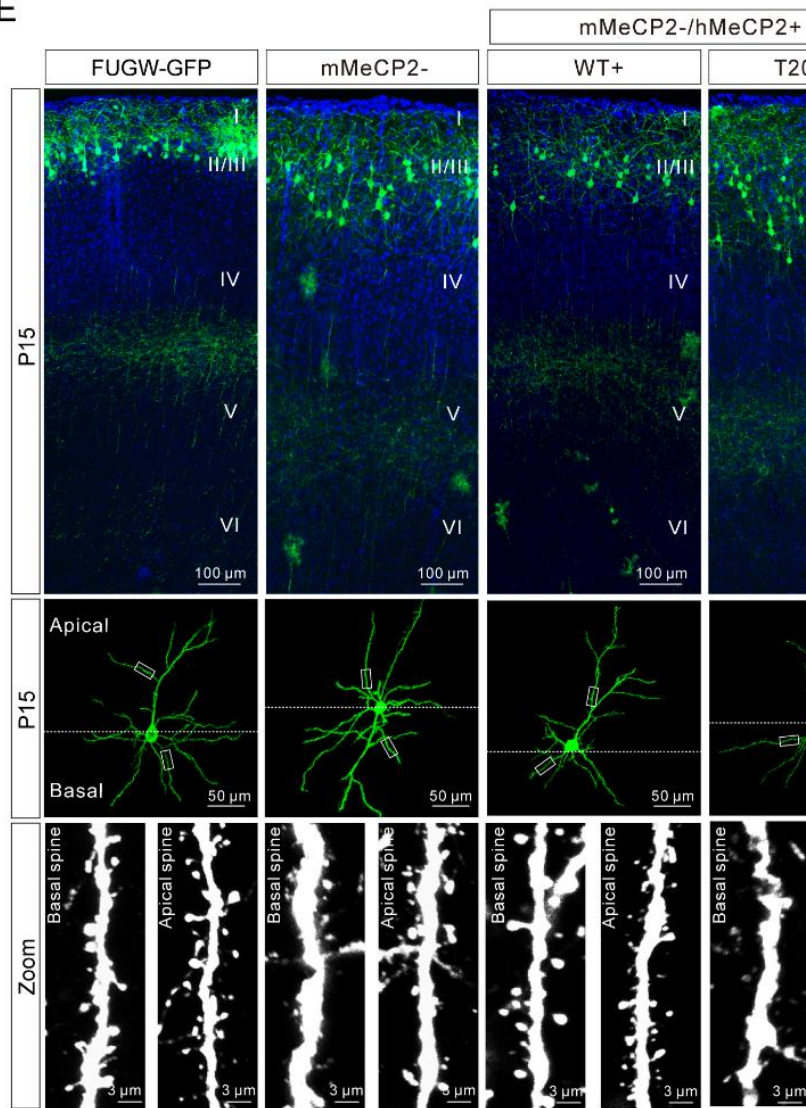

$\mathrm{F}$

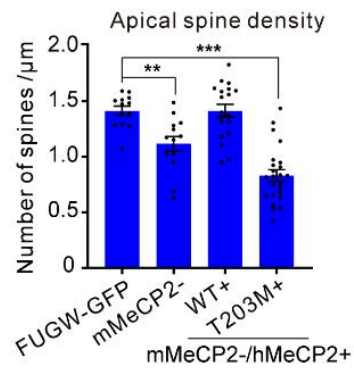

G

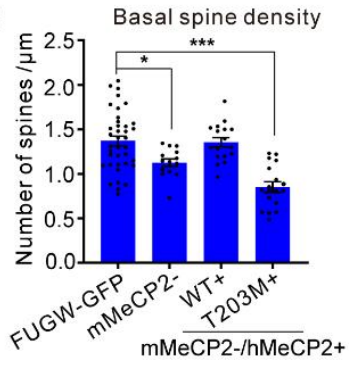

$\mathrm{H}$

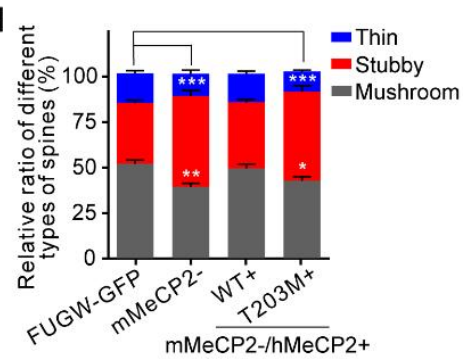

Figure 5. T203 O-GIcNAcylation is Required for Dendritic Spine Morphogenesis in vivo 
bioRxiv preprint doi: https://doi.org/10.1101/2020.06.17.158311; this version posted June 18, 2020. The copyright holder for this preprint (which was not certified by peer review) is the author/funder. All rights reserved. No reuse allowed without permission.

A

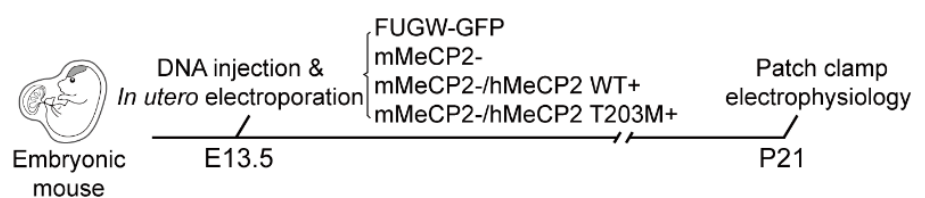

B

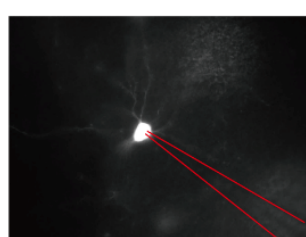

$\mathrm{E}$

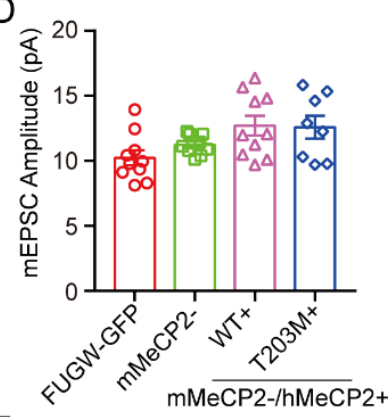

$\mathrm{F}$
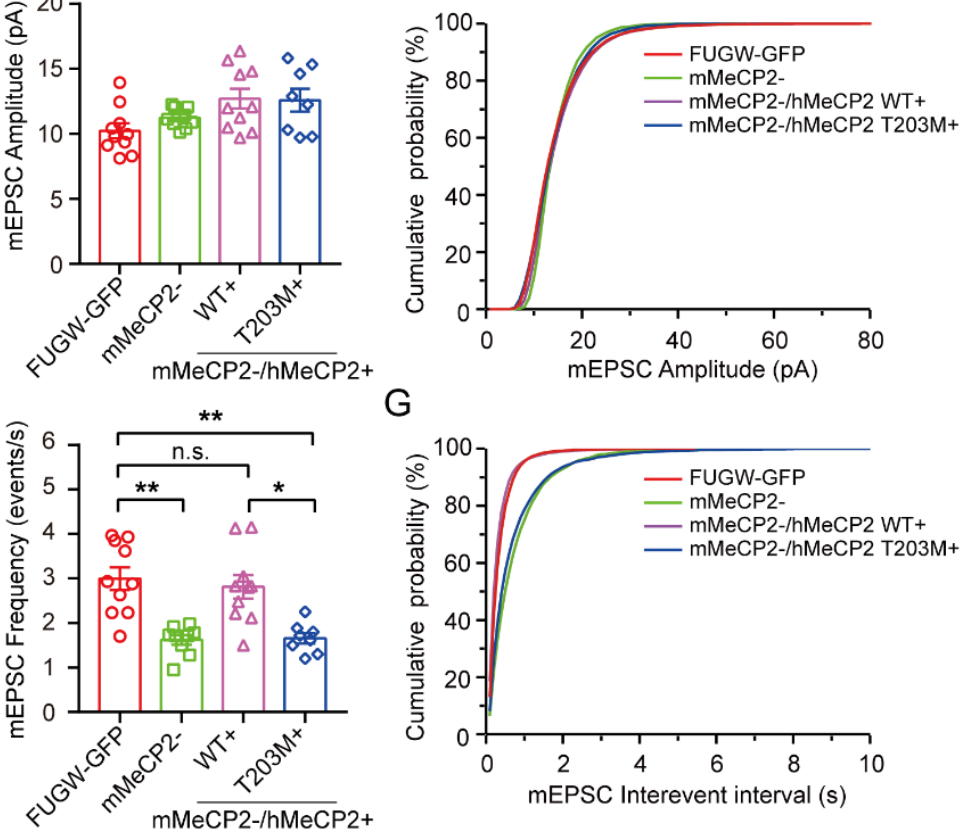

G

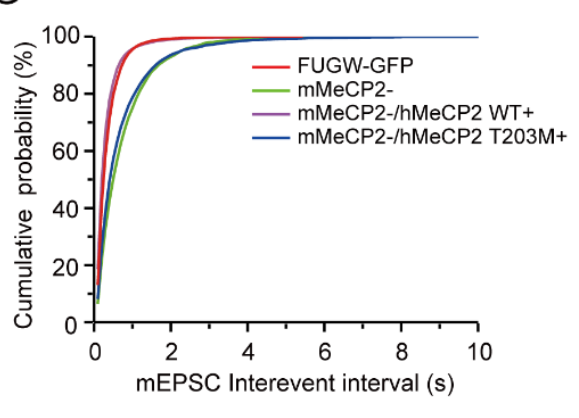

$\mathrm{H}$

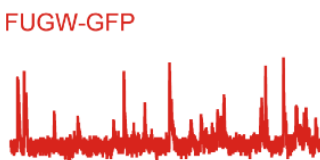

mMeCP2-

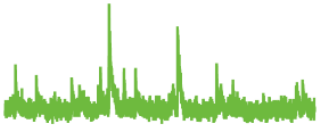

1

$\mathrm{K}$

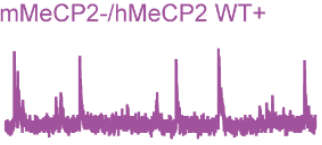

mMeCP2-/hMeCP2 T203M+

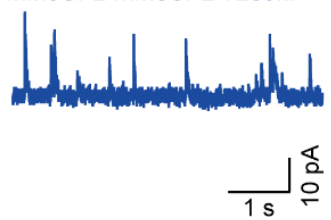

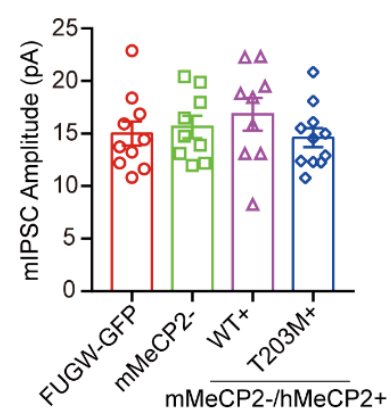
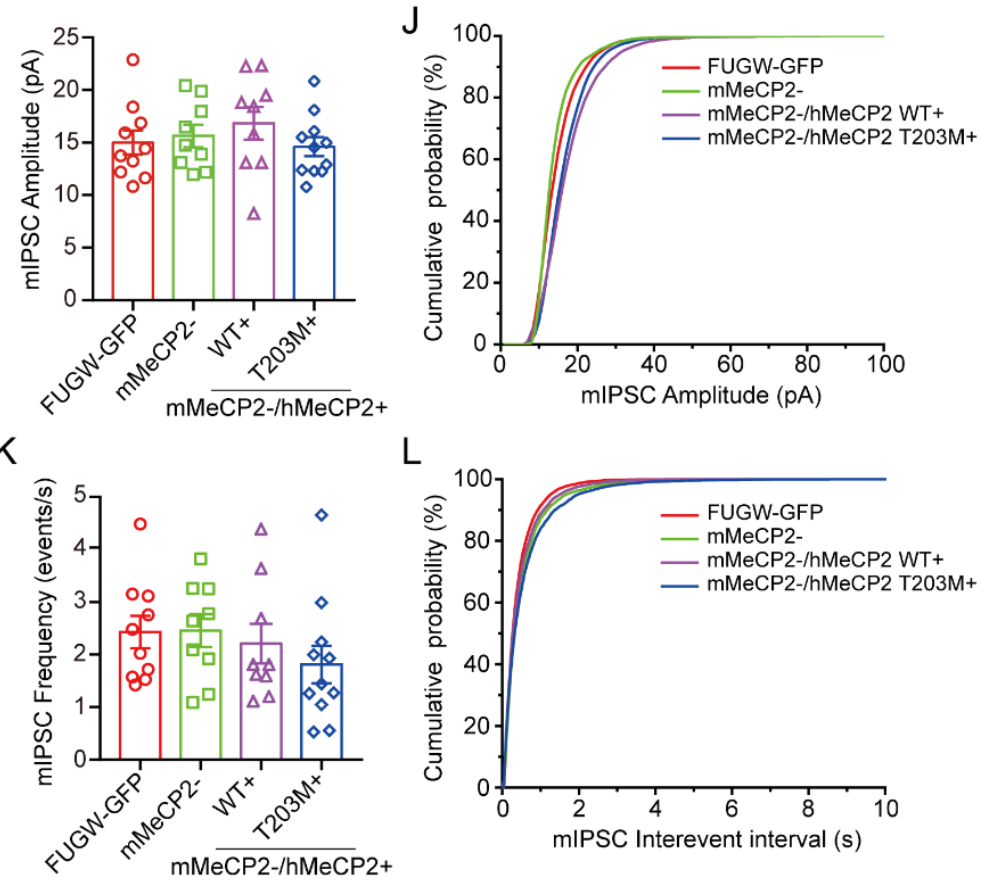

$\mathrm{L}$

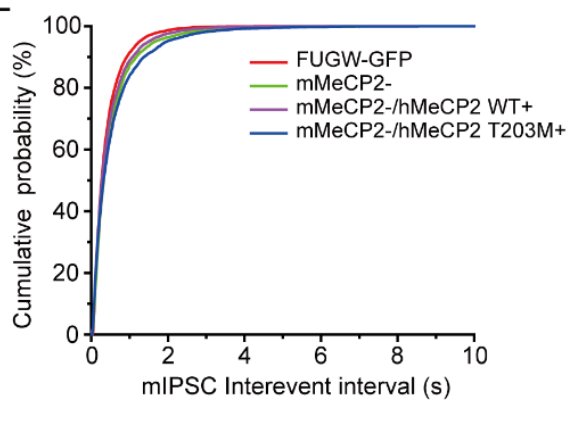

Figure 6. T203 O-GIcNAcylation is Essential for the Excitatory Synaptic Transmission in the Neocortex 
bioRxiv preprint doi: https://doi.org/10.1101/2020.06.17.158311; this version posted June 18, 2020. The copyright holder for this preprint (which was not certified by peer review) is the author/funder. All rights reserved. No reuse allowed without permission.

A

FUGW-GFP

mMeCP2-

$\mathrm{mMeCP} 2-/ \mathrm{hMeCP} 2+$

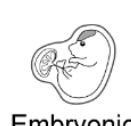

corticalion of Primary Lentivirus

mMeCP2-/hMeCP2 T203M

cortical neurons culture infection mMeCP2-/hMeCP2 T436A+

$1 \mu \mathrm{M}$ TTX $55 \mathrm{mM} \mathrm{KCl}$ RNA mMeCP2-/hMeCP2-2Muts+

$\begin{array}{ccc}/ & / \\ \text { E15.5 DIV } 0 & \text { DIV } 3\end{array}$

overnight treatment extraction Q-PCR

$\mathrm{B} \quad \mathbf{E}_{+\mathrm{KCl}}^{-\mathrm{KCl} C}$

$\mathrm{C}=-\mathrm{KCl}$

$\mathrm{D}-\mathrm{KCl} E$

DIV $85 \mathrm{hr}$ later

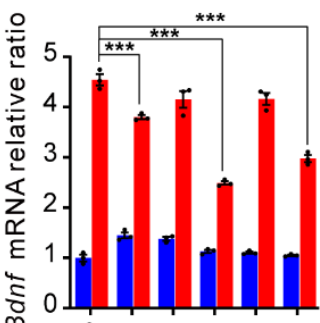

CPPQ $202^{x} N^{x} 6 R^{x}+s^{x}$

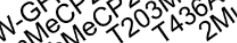

mMeCP2-
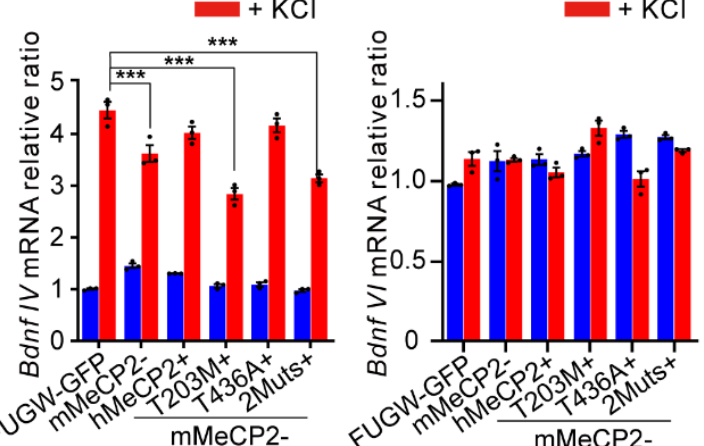

$\mathrm{E}=-\mathrm{KCl}$
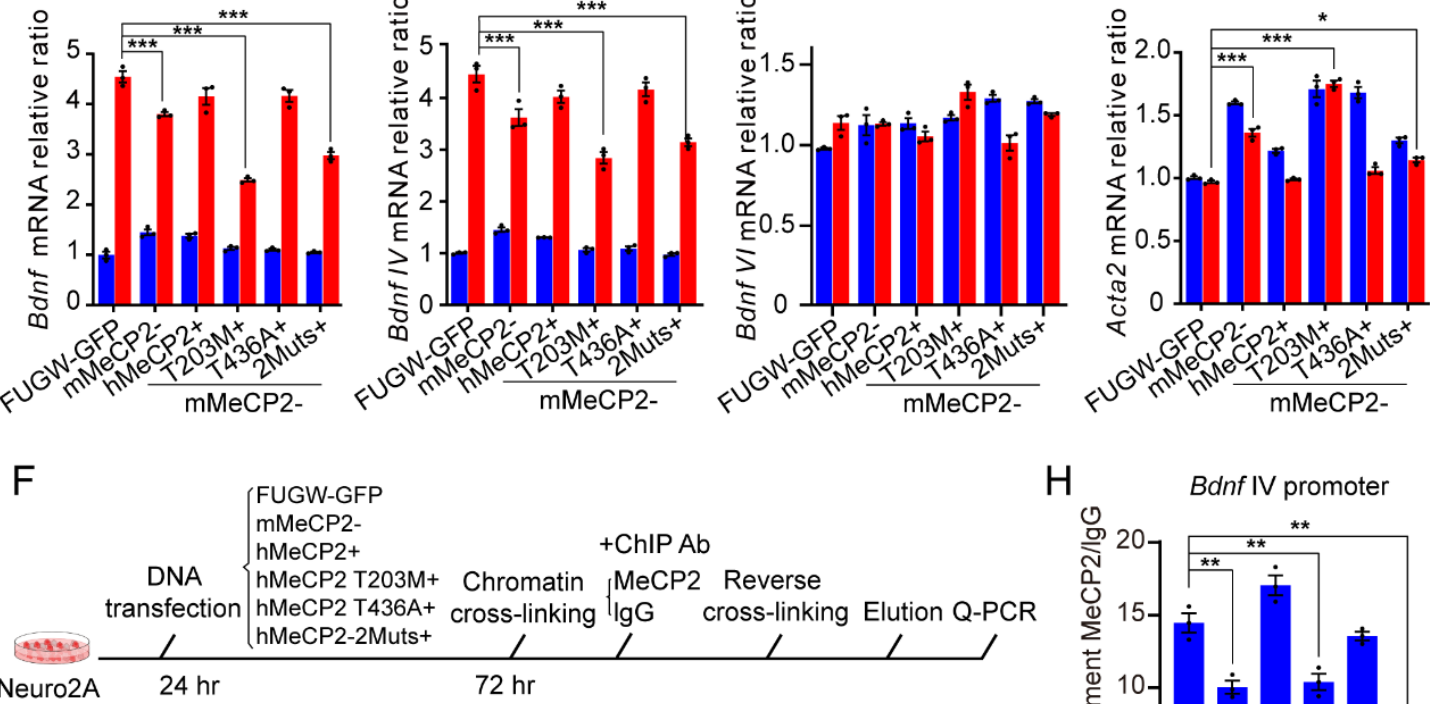

G
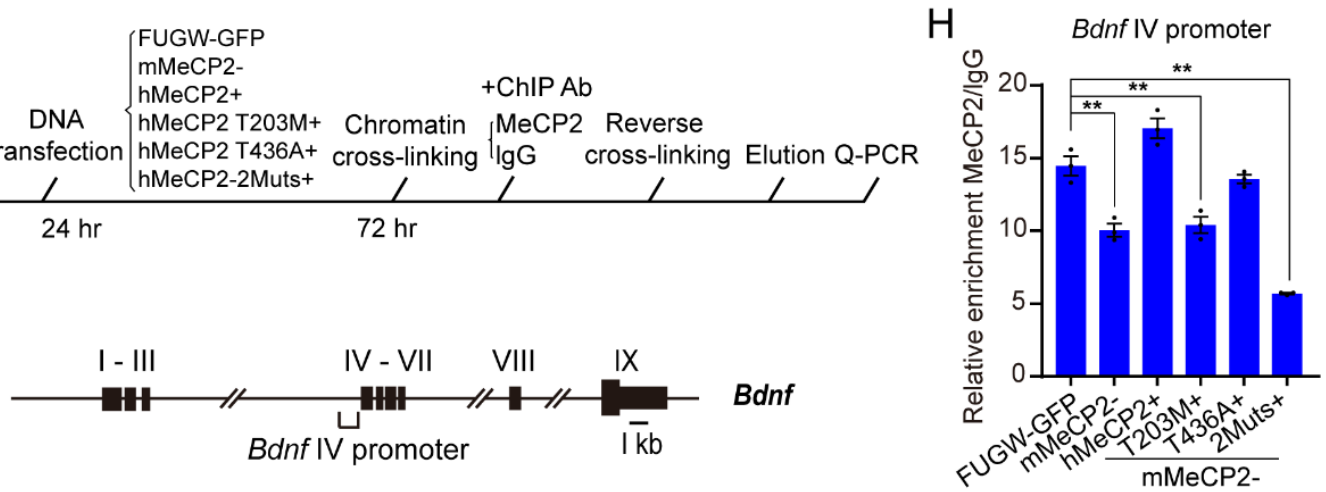

Figure 7. T203 O-GIcNAcylation Activates Bdnf Promoter IV-Dependent Transcription 